\title{
Anticancer immunotherapy by CTLA-4 blockade: obligatory contribution of IL-2 receptors and negative prognostic impact of soluble CD25
}

Dalil Hannani ${ }^{1,2,3, *}$, Marie Vétizou ${ }^{1,2,3, *}$, David Enot ${ }^{1,4,5}$, Sylvie Rusakiewicz ${ }^{1,2,6}$, Nathalie Chaput ${ }^{1,2,6}$, David Klatzmann ${ }^{7,8}$, Melanie Desbois ${ }^{1,2,6}$, Nicolas Jacquelot ${ }^{1,2,3}$, Nadège Vimond ${ }^{1,2,6}$, Salem Chouaib ${ }^{9}$, Christine Mateus ${ }^{1,10}$, James P Allison ${ }^{11}$, Antoni Ribas ${ }^{12}$, Jedd D Wolchok ${ }^{13}$, Jianda Yuan ${ }^{13}$, Philip Wong ${ }^{13}$, Michael Postow $^{13}$, Andrzej Mackiewicz ${ }^{14,15}$, Jacek Mackiewicz ${ }^{14,15}$, Dirk Schadendorff ${ }^{16}$, Dirk Jaeger ${ }^{17}$, Alan J Korman $^{18}$, Keith Bahjat ${ }^{19}$, Michele Maio ${ }^{20}$, Luana Calabro ${ }^{20}$, Michele WL Teng ${ }^{21,22}$, Mark J Smyth ${ }^{21,22}$, Alexander Eggermont ${ }^{1,2,23}$, Caroline Robert ${ }^{1,2,10,23}$, Guido Kroemer ${ }^{1,4,5,24,25,26}$, Laurence Zitvogel ${ }^{1,2,3,10}$

${ }^{I}$ Gustave Roussy Cancer Campus, Villejuif, France; ${ }^{2}$ INSERM U1015, Villejuif, France; ${ }^{3}$ Université Paris Sud-XI, Faculté de Médecine, Le Kremlin Bicêtre, France; ${ }^{4}$ INSERM U848, Villejuif, France; ${ }^{5}$ Metabolomics Platform, Institut Gustave Roussy, Villejuif, France; ${ }^{6}$ Center of Clinical Investigations in Biotherapies of Cancer (CICBT) 507, Villejuif, France; ${ }^{7}$ INSERM, Unité Mixte de Recherche_S959, $I^{3}$, Paris, France; ${ }^{8}$ Assistance-Publique Hopitaux de Paris, Hôpital Pitié-Salpêtrière, Biotherapy, Paris, France;

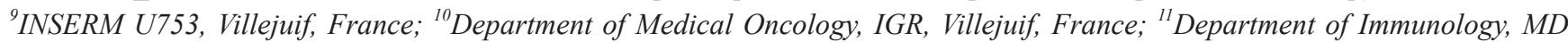
Anderson Cancer Center, Houston, TX, USA; ${ }^{12}$ University of California Los Angeles, Los Angeles, CA, USA, ${ }^{13}$ Ludwig Center for Cancer Immunotherapy, Department of Immunology, and Department of Medicine, Memorial Sloan-Kettering Cancer Center, New York, NY, USA; ${ }^{14}$ Department of Diagnostics and Cancer Immunology, Greater Poland Cancer Centre, Poznan, Poland; ${ }^{15}$ Chair of Medical Biotechnology, Poznan University of Medical Sciences, Poznan, Poland; ${ }^{16}$ Department of Dermatology, University Hospital Essen, Germany; ${ }^{17}$ National Center for Tumor Diseases, University Hospital, Heidelberg, Germany; ${ }^{18}$ Bristol-Myers Squibb, Redwood City, CA, USA; ${ }^{19}$ Earle A Chiles Research Institute, Robert W. Franz Cancer Center, Providence Portland Medical Center, Portland, OR, USA, ${ }^{20}$ Medical Oncology and Immunotherapy, Department of Oncology, University Hospital of Siena, Istituto Toscano Tumori, Siena, Italy; ${ }^{21}$ QIMR Berghofer Medical Research Institute, Herston, 4006, Queensland, Australia; ${ }^{22}$ School of Medicine, The University of Queensland, Herston, QLD 4006, Queensland, Australia; ${ }^{23}$ INSERM U981, Villejuif, France; ${ }^{24}$ Equipe 11 labellisée Ligue contre le Cancer, Centre de Recherche des Cordeliers, INSERM U 1138, Paris, France; ${ }^{25}$ Pôle de Biologie, Hôpital Européen Georges Pompidou, AP-HP, Paris, France, ${ }^{26}$ Université Paris Descartes, Sorbonne Paris Cité, Paris, France

The cytotoxic T lymphocyte antigen-4 (CTLA-4)-blocking antibody ipilimumab induces immune-mediated longterm control of metastatic melanoma in a fraction of patients. Although ipilimumab undoubtedly exerts its therapeutic effects via immunostimulation, thus far clinically useful, immunologically relevant biomarkers that predict treatment efficiency have been elusive. Here, we show that neutralization of IL-2 or blocking the $\alpha$ and $\beta$ subunits of the IL-2 receptor (CD25 and CD122, respectively) abolished the antitumor effects and the accompanying improvement of the ratio of intratumoral $T$ effector versus regulatory cells (Tregs), which were otherwise induced by CTLA-4 blockade in preclinical mouse models. CTLA-4 blockade led to the reduction of a suppressive $\mathrm{CD}^{+} \mathrm{T}$ cell subset expressing Lag3, ICOS, IL-10 and Egr2 with a concomitant rise in IL-2-producing effector cells that lost FoxP3 expression and accumulated in regressing tumors. While recombinant IL-2 improved the therapeutic efficacy of CTLA-4 block-

*These two authors contributed equally to this work.

Correspondence: Laurence Zitvogel ${ }^{\mathrm{a}}$, Guido Kroemer $^{\mathrm{b}}$

${ }^{a}$ E-mail: laurence.zitvogel@gustaveroussy.fr

${ }^{b}$ E-mail: kroemer@orange.fr

Abbreviations: CTLA-4 (cytotoxic T lymphocyte antigen-4); MM (metastatic melanoma); MoA (mode of action); Lag3 (lymphocyte-activation gene 3); ICOS (inducible T-cell costimulator); ICOSL (ICOS ligand); Egr-2 (early growth response gene-2); rIL-2 (recombinant interleukin 2); sCD25 (soluble CD25 (IL-2 receptor alpha chain)); Th (T helper); GM-
CSF (granulocyte-macrophage colony-stimulating factor); GVAX (GMCSF gene-transfected tumor cell vaccine); Treg (regulatory T cell); Teff (effector T cell); MICA (MHC class I-related chain A); IFNAR (interferon- $\alpha / \beta$ receptor); R (responder); NR (non responder); OS (overall survival); ir-RC (immune response-related criteria); TCR (T-cell receptor); TILs (tumor-infiltrating lymphocytes);

Received 22 May 2014; revised 13 August 2014; accepted 11 September 2014; published online 13 January 2015 
ade, the decoy IL-2 receptor $\alpha$ (IL-2R $\alpha$, sCD25) inhibited the anticancer effects of CTLA-4 blockade. In 262 metastatic melanoma patients receiving ipilimumab, baseline serum concentrations of sCD25 represented an independent indicator of overall survival, with high levels predicting resistance to therapy. Altogether, these results unravel a role for IL-2 and IL-2 receptors in the anticancer activity of CTLA-4 blockade. Importantly, our study provides the first immunologically relevant biomarker, namely elevated serum SCD25, that predicts resistance to CTLA-4 blockade in patients with melanoma.

Keywords: ipilimumab; CTLA-4 blockade; IL-2; CD122; sCD25; tumor immunotherapy; cancer

Cell Research (2015) 25:208-224. doi:10.1038/cr.2015.3; published online 13 January 2015

\section{Introduction}

The concept of cancer immune surveillance and editing, originally coined by Burnet and Thomas and then proven by Schreiber's group in mice [1], paved the way to the clinical demonstration of the relevance of intratumoral effector memory Th1 or Tc1 cells to the prognosis of human malignancies [2]. Measures designed to induce cellular anticancer immune responses and to promote the influx of specific $\mathrm{T}$ lymphocytes into tumor beds may constitute a valid strategy for prolonging patient survival. Multiple T cell co-stimulatory and co-inhibitory receptors act in concert to regulate the activation, proliferation and effector functions of T lymphocytes. CD28 and CTLA-4 are among the best characterized T cell co-stimulatory and co-inhibitory molecules, respectively [3]. Both molecules are expressed on the surface of selected $\mathrm{T}$ populations and transmit opposing signals upon interaction with their ligands, CD80 (B7-1) and CD86 (B7-2) on antigen-presenting cells [3].

The CTLA-4 blocking antibody ipilimumab was approved by the US Food and Drug Administration (FDA) and the European Medical Agency (EMA) in 2011 for the treatment of advanced metastatic melanoma (MM) after the publication of two phase III clinical trials showing its capacity to prolong overall survival of patients with advanced MM $[4,5]$. However, only a fraction of MM patients exhibited a long-term benefit from ipilimumab treatment. This fact, coupled to the close-to-prohibitive costs and major side effects of this therapeutic regimen, has spurred interest in the discovery of biomarkers that would predict the efficacy of ipilimumab. A number of preclinical studies have defined (some of) the basic mechanisms underlying the activity of this first-in-class product. Blocking the interaction between CTLA-4 and its known ligands B7-1 and B7-2 reverses intrinsic and extrinsic inhibitory effects of CTLA-4 on T cell functions $[6,7]$, thus leading to enhanced $\mathrm{T}$ cell proliferation, cytokine production and intratumoral accumulation of effector $\mathrm{CD}^{+}$and $\mathrm{CD} 8^{+}$T cells. CTLA-4 inhibits inter- leukin-2 (IL-2) transcription in $\mathrm{CD}^{+} \mathrm{T}$ lymphocytes [6, $8]$, which is likely due to its ability to stimulate TGF- $\beta$ release by $\mathrm{CD}^{+} \mathrm{T}$ cells [9]. Moreover, knockout or neutralization of CTLA-4 inhibits Treg function [10, 11], and antibody-dependent cell-mediated cytotoxicity (ADCC) or opsonization mediated by anti-CTLA-4 antibodies may favor the elimination of intratumoral regulatory $\mathrm{T}$ cells (Tregs) [12]. CTLA-4 blockade improves the ratio of intratumoral effector $\mathrm{T}$ cells to Tregs, when combined with tumor vaccines $[13,14]$, indoleamine 2,3-dioxygenase (IDO) inhibitors [15] or chemotherapeutic agents [16].

Several pharmacodynamic markers of ipilimumab bioactivity have been reported. In MM patients, ipilimumab treatment increased the absolute lymphocyte count [17] and the frequencies of circulating $\mathrm{HLA}-\mathrm{DR}^{+}$or $\mathrm{ICOS}^{+}$ $\mathrm{T}$ cells $[18,19]$. An increase in the absolute eosinophil count has also been associated to clinical benefit [17]. Yuan et al. [20] showed multifunctional $\mathrm{T}$ and $\mathrm{B}$ cell responses directed against the prominent tumor antigen NY-ESO-1 in a fraction of responding patients. The ratio of tumor-infiltrating $\mathrm{CD}^{+} \mathrm{T}$ effector cells to $\mathrm{FoxP}^{+}$ Tregs after ipilimumab infusion was tightly correlated with the extent of tumor shrinkage in MM and ovarian cancer patients $[18,21]$. This correlation between a high $\mathrm{CD}^{+} \mathrm{T}$ effector/Treg ratio and tumor regression has been observed in patients treated with the human IgG1 ipilimumab (which mediates ADCC) but less frequently in those treated with the IgG2 tremelimumab [22, 23]. $\mathrm{CD}^{+}{ }^{+} \mathrm{ICOS}^{\text {high }} \mathrm{IFN} \gamma^{+} \mathrm{T}$ cells recognizing tumor epitopes were found after radical prostatectomy in prostate cancers and non-malignant tissues post-neoadjuvant ipilimumab [24]. Finally, correlations between therapy-induced antibody responses and tumor regression have been reported in advanced ovarian and melanoma patients. Dranoff's group reported that patients responding to GM-CSF-expressing tumor cell-based vaccine (GVAX) treatment followed by ipilimumab infusions generated high titers of antibodies against MHC class I-related chain A (MICA) that antagonized the immune suppression evoked by soluble MICA and stimulated innate and 
adaptive antitumor cytotoxicity [25].

CTLA-4 blockade in cancer might unleash preexisting or vaccine-induced antitumor effector and/or memory $\mathrm{T}$ cell activities from inhibition. Thus, anti-CTLA-4 may function best in the subset of patients with high levels of tumor-infiltrating lymphocytes (TILs) and intratumoral Tregs $[22,26]$, low serum level of VEGF [27] and preexisting responses to tumor antigens such as NY-ESO-1 [20]. However, large prospective studies demonstrating the clinical utility of baseline markers that can predict CTLA-4 blockade effectiveness (as opposed to a pharmacokinetic/dynamic parameters) are still ongoing.

Driven by these considerations, we explored the mechanism underlying the action of CTLA-4 blockade, hoping to identify clinically useful biomarkers that predict the treatment efficiency. We focused on the IL-2/IL-2 receptor (IL2R) system due to its potential role in the action of ipilimumab. Indeed, one study performed in mice revealed an essential role of IL-2 in mediating the antitumor activity of CTLA-4 blockade [28]. Moreover, a clinical trial showed that the therapeutic effects of CTLA-4 blockade against melanoma were not improved by simultaneous treatment with recombinant IL-2 (rIL-2) [29], suggesting that both treatments may act through the same pathway. Our results here uncover a key role for IL-2 and several components of the IL-2R complex (CD25 and CD122) in the antitumor effects of anti-CTLA-4 antibodies in murine colon cancer and sarcoma models. Neutralization of CD122 abolished the effects of CTLA4 blockade in elevating intratumoral $\mathrm{CD}^{+}$and $\mathrm{CD}^{+} \mathrm{T}$ effector/Treg ratios. The main source of IL-2 was the immunosuppressive $\mathrm{CD}^{+} \mathrm{T}$ cell subset expressing Lag3, Egr2, IL-10, ICOS and FoxP3, which was converted into or was replaced by an IL-2-producing FoxP3-negative $\mathrm{T}$ cell population accumulating in tumor beds upon blockade of mouse CTLA-4 (mCTLA-4). Hence, the efficacy of mCTLA-4 blockade was significantly potentiated by rIL-2 or anti-IL-10R and anti-Lag3 (but not ICOS) neutralizing antibodies in mice. A single infusion of ipilimumab triggered an increase in circulating levels of soluble CD25 (sCD25) and Lag3 in MM patients. High levels of sCD25, which was reported to act as a decoy receptor [30, 31], were detrimental for the anticancer activity of anti-mCTLA- $4 \mathrm{Ab}$ and ipilimumab in mice and patients, respectively. The best multivariate model calculated based on nine cohorts of $262 \mathrm{MM}$ patients suggests that individuals with $\mathrm{sCD} 25^{\text {high }} \mathrm{LDH}^{\text {high }}$ serum signatures at diagnosis are less likely to benefit from ipilimumab therapy than those with low pre-treatment levels of sCD25.

\section{Results}

IL-2/IL-2R $\alpha \beta$-dependent antitumor effects of anti-mCT-

\section{LA-4 therapy}

Several cytokine-related signaling pathways may affect the host-tumor equilibrium in advanced human malignancies [32-34]. We therefore investigated the putative contribution of two critical cytokine receptors, the IL-2R and the type I interferon receptor (IFNAR1), to the antitumor effects of CTLA4 blockade. These effects were evaluated in two preclinical models, MCA205 sarcomas and MC38 colon cancers that were both engineered to express the model antigen ovalbumin (OVA). While IFNAR1 was dispensable for the anticancer activity of anti-mCTLA-4 Ab (Supplementary information, Figure S1A), components of the IL-2R-related signaling pathway were required for the antitumor effects of mCTLA-4 blockade (Figure 1A-1D). Antibody-mediated neutralization of the two interleukins that bind to the IL-2R $\beta \gamma$ complex, IL-2 and IL-15, as well as that of two IL-2R subunits, IL-2R $\alpha(\mathrm{CD} 25)$ and IL-2R $\beta$ (CD122), reduced the efficacy of mCTLA-4 blockade in both tumor models (except for the effects of anti-CD25, which were only examined in the MCA205-OVA model; Figure 1A-1D). Blockade of the IL-2R $\alpha$ chain was accompanied by the loss of effector $\mathrm{CD} 4^{+} \mathrm{CD} 25^{+} \mathrm{FoxP} 3^{-}$lymphocytes (data not shown). IL-2 is produced by activated $\mathrm{T}$ cells and acts on $\mathrm{T}$ and NK cells to amplify their proliferation and effector functions [35]. In both tumor models, we found that mCTLA-4 blockade lost its tumor growth-reducing effects upon depletion of $\mathrm{CD}^{+} \mathrm{T}$ cells (Supplementary information, Figure $\mathrm{S} 1 \mathrm{~B}$ ) or $\mathrm{CD} 8^{+} \mathrm{T}$ cells (Supplementary information, Figure S1C) but not upon depletion of NK cells using NK1.1-specific or asialo-GM1-specific antibodies or upon blockade of NKG2D (Supplementary information, Figure S1D-S1F).

We next analyzed possible biomarkers predicting the efficacy of mCTLA-4 blockade in the MCA205-OVA model. For this purpose, we segregated animals exhibiting tumor stabilization after 3 inoculations of anti-mCTLA-4 Ab (noted as responders, R) from those that failed to respond (noted as non-responders, NR) (Figure 2A, left panel). At the time of sacrifice, the inhibitory effects of an anti-CD122 antibody on the efficacy of mCTLA-4 blockade were already apparent (Figure 2A, right panel). Flow cytometric analyses of tumor-infiltrating cells revealed that CTLA-4 blockade (i) induced an influx of leukocytes in $\mathrm{R}$ but not in NR mice (data not shown), (ii) increased the frequency of intratumoral IFN $\gamma^{+} \mathrm{CD} 4^{+}$ lymphocytes in R (Figure 2B, left panel), and (iii) increased the ratio of $\mathrm{CD}^{+} / \mathrm{CD}^{+}{ }^{+} \mathrm{FoxP} 3^{+} \mathrm{T}$ cells in $\mathrm{R}$ (Figure $2 \mathrm{C}$, left panel). These two latter effects were fully reversed upon blockade of IL-2R $\beta$ (Figure 2B-2C, right panels). Contrasting with splenic $\mathrm{CD}^{+} \mathrm{FoxP}^{+}$Tregs, which increased in number after CTLA-4 blockade (Sup- 
A

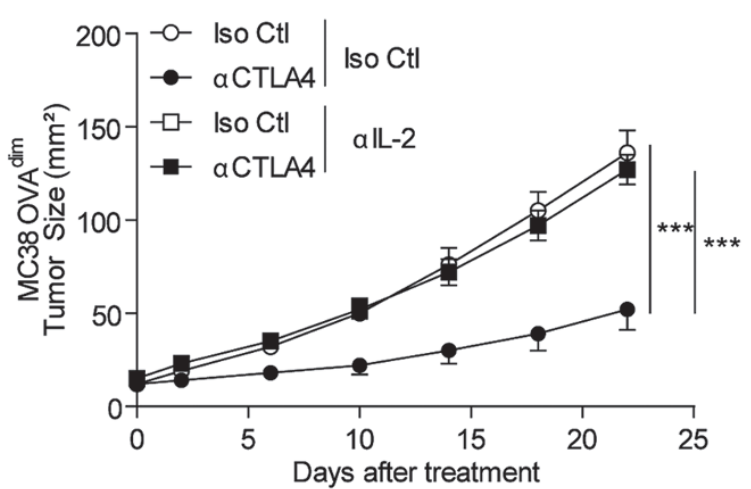

B

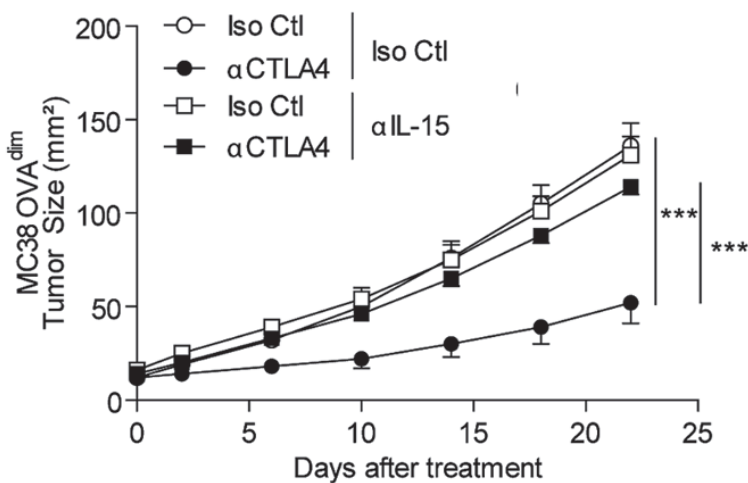

C

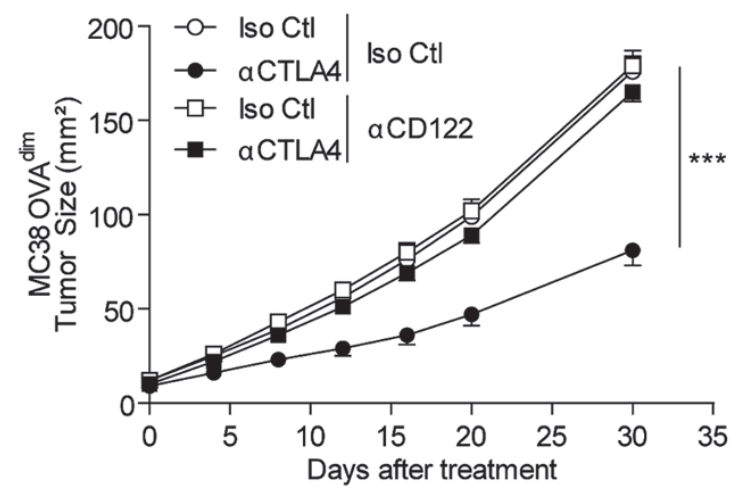

D

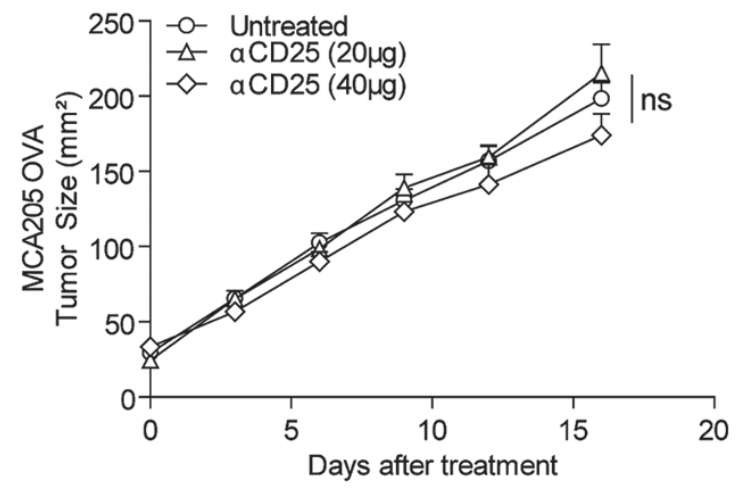

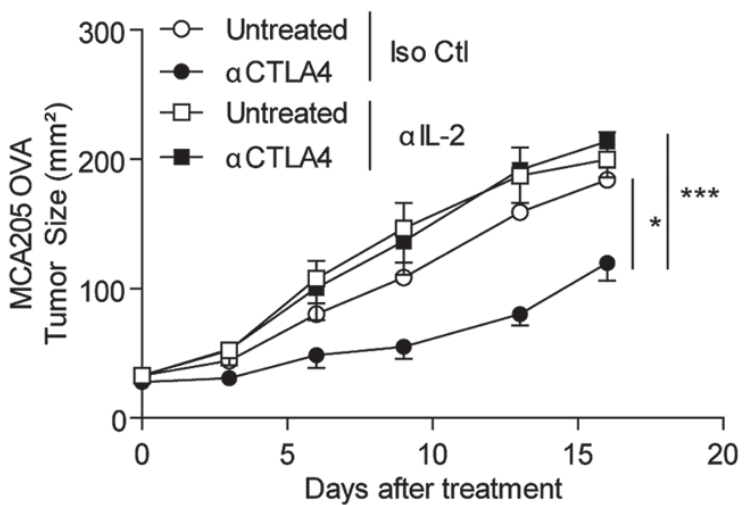
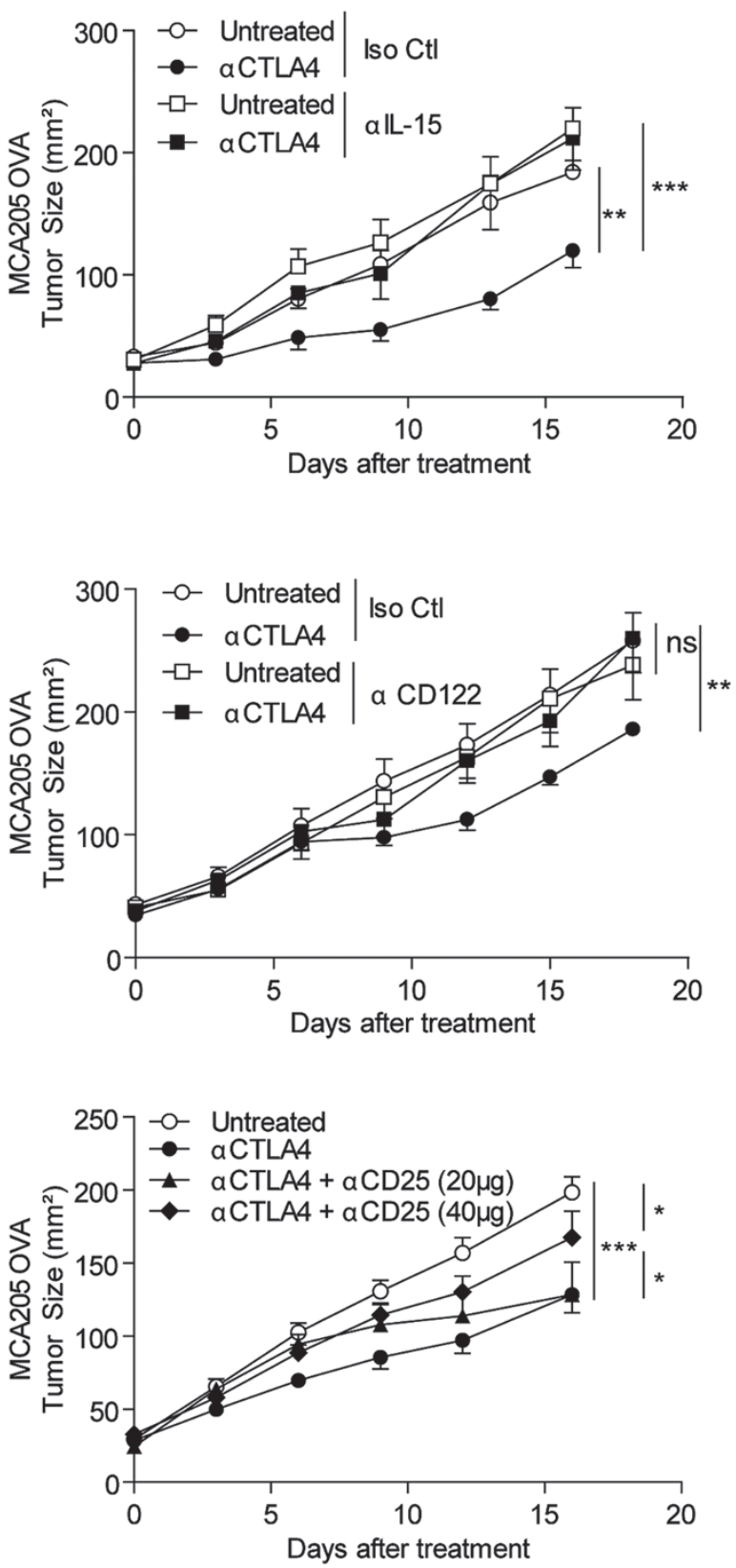
plementary information, Figure S2A), the frequency of intratumoral Tregs strongly diminished in $\mathrm{R}$ and to a lesser extent in NR mice (Supplementary information, Figure S2B). However, ICOS expression was upregulated in $\mathrm{CD}^{+}$TILs in R mice (Figure 2D, left panel) independently of CD122 (Figure 2D, right panel), paralleling the loss of ICOSL expression on myeloid CD $11 \mathrm{~b}^{+} \mathrm{CD} 11 \mathrm{c}^{+}$ cells (data not shown).

From these data, we conclude that IL-2 and its high-affinity receptor are both critical for $\mathrm{T}$ cell-dependent control of tumor progression induced by mCTLA-4 blockade.

$C D 4^{+} \mathrm{Lag}^{+} \mathrm{T}$ cells showed upregulated IL-2 transcription upon CTLA-4 blockade

In accord with the implication of endogenous IL-2 in the action of CTLA4 blockade, and consistent with a previous work [28], rIL-2 greatly synergized with the mCTLA-4 Ab in terms of reducing tumor size (Figure 3A, left panel) and increasing circulating $\mathrm{CD}^{+} \mathrm{T}$ lymphocyte numbers (Figure 3A, middle panel). Surprisingly, we observed an intratumoral accumulation of a subset of $\mathrm{CD} 4{ }^{+}$ $\mathrm{T}$ lymphocytes expressing high levels of Lag $3^{+}$(Figure $3 \mathrm{~A}$, right panel). This unexpected finding prompted us to perform a comprehensive phenotypic analysis of $\mathrm{CD} 4{ }^{+} \mathrm{Lag} 3^{+} \mathrm{T}$ cells before and after mCTLA4 blockade.

The number of a small subset of $\mathrm{CD}^{+} \mathrm{T}$ cells defined by membrane expression of Lag3 was increased in the spleen of $R$ versus NR mice (Figure $3 B$, left panel). Their number in tumor beds was higher in $\mathrm{R}$ than NR mice (Figure $3 \mathrm{~B}$, right panel). In contrast to $\mathrm{CD} 4^{+} \mathrm{Lag} 3$ $\mathrm{T}$ cells, $\mathrm{CD} 4^{+} \mathrm{Lag} 3^{+} \mathrm{T}$ cells showed downregulated ICOS expression $(45 \% \pm 10 \%$ pre- versus $20 \% \pm 5 \%$ post-anti-mCTLA-4 Ab, data not shown) and FoxP3 expression $(45 \% \pm 20 \%$ pre- versus $15 \% \pm 10 \%$ post-anti-mCTLA- 4 $\mathrm{Ab}$, Figure 3C), which were more evident in R than NR tumors, leading to the maintenance of $\mathrm{CD}^{+}{ }^{+} \mathrm{Lag} 3^{+} \mathrm{FoxP} 3$ cells in tumors upon CTLA-4 blockade. Next, we performed flow cytometry-guided cell sorting of various TIL and splenic subsets after staining with Abs specific for CD4, CD8, ICOS, Lag3, CD25 or CD127 to analyze the transcription of $I L-2$. While splenic non-Treg CD4 $\mathrm{T}$ cells from mice treated with anti-mCTLA- 4 antibody produced IL-2 (5-fold increase) regardless of Lag3 expression (Figure 3D, left panel), intratumoral $\mathrm{CD} 4^{+} \mathrm{Lag} 3^{+}$ $\mathrm{T}$ cells showed selectively and markedly upregulated IL-2 transcription (by $\sim 15$-fold) upon mCTLA-4 blockade (Figure 3D, right panel). Concomitantly, transcription of the immunosuppressive products that represent hallmarks of $\mathrm{CD} 4^{+} \mathrm{Lag} 3^{+}$cells, such as IL-10 and Egr-2 [36], slightly decreased after mCTLA-4 blockade (Figure 3E). The simultaneous blockade of CTLA- 4 and IL-10R or that of CTLA-4 and Lag3 had additive tumor growth-inhibitory effects (Figure 3F, left and middle panels), while ICOS inhibition failed to improve the therapeutic effects of the anti-mCTLA-4 Ab (Figure 3F, right panel).

Altogether, CTLA-4 blockade alters the functional profile of $\mathrm{CD} 4^{+} \mathrm{Lag} 3^{+} \mathrm{T}$ cells, which become the major source of intratumoral IL-2. At present, it is not clear whether this results from their phenotypic conversion or may be explained by the replacement within tumor beds of one $\mathrm{T}$ cell population by another that lacks FoxP3 expression and produces IL-2.

\section{sCD25 inhibits the efficacy of CTLA-4 blockade}

We next monitored levels of surrogate markers of lymphocyte activation such as soluble CD25 (sCD25) and Lag3 (sLag3) in the serum of MM patients treated with ipilimumab. Similar to patients with autoimmune vasculitis receiving low-dose rIL-2 [37], MM patients ( $n$ $=262$ ) treated with ipilimumab (most of whom received $3 \mathrm{mg} / \mathrm{kg}$ on a compassionate basis, Supplementary information, Table S1) and patients with an autoimmune disease $(n=9)$ treated with low-dose rIL-2 exhibited a significant rise in their serum sCD25 levels (Figure 4A4B), as well as, though to a lesser extent, serum sLag3 levels (Figure 4C-4D). Similar results were obtained from a cohort of $20 \mathrm{MM}$ patients treated with the alternative anti-CTLA-4 Ab, tremelimumab (3 weeks after a single dose of $15 \mathrm{mg} / \mathrm{kg}$ ) (Figure 4E). Intriguingly, a

Figure 1 IL-2/IL-2R $\alpha \beta$-dependent antitumor effects of mCTLA-4 blockade. Three intraperitoneal (i.p.) injections of anti-mCTLA-4 Ab (clone 9D9) were administered every 3 days to treat MC38-OVA colon cancers (A-C, left panels) or MCA205-OVA sarcomas (A-C, right panels) once they had reached a size of 25-30 $\mathrm{mm}^{2}$. (A-B) Role of IL-2 and IL-15 in tumor growth control by CTLA-4 blockade. Neutralizing antibodies against IL-2 (A) or IL-15 (B) were injected intratumorally at the same time when anti-CTLA-4 was administered into mice bearing MC38-OVA ${ }^{\text {dim }}$ (left panels) or MCA205-OVA (right panels) tumors. (C) Role of CD122 in tumor growth control by CTLA-4 blockade. Anti-CD122 neutralizing Ab was injected i.p. One day before each anti-CTLA-4 treatment. (D) Role of CD25 during anticancer therapy with anti-CTLA-4 antibodies. Idem as in A but anti-CD25 mAb was co-administered at a total dose of 20 or $40 \mu \mathrm{g}$ intratumorally on days 0,3 and 6 in MCA205-OVA. Groups untreated or treated with anti-CD25 alone are shown in the left panel, while the combined effects of anti-CD25 and anti-CTLA-4 are shown in the right panel. Tumor growth kinetics was depicted for one representative experiment (5-6 mice/group) out of 2-4 experiments yielding similar results. Tumor size is shown as mean \pm SEM. ${ }^{*} P<0.05,{ }^{* *} P<0.01,{ }^{* *} P<0.001$, ns, not significant (Student's $t$ test or ANOVA). 
A

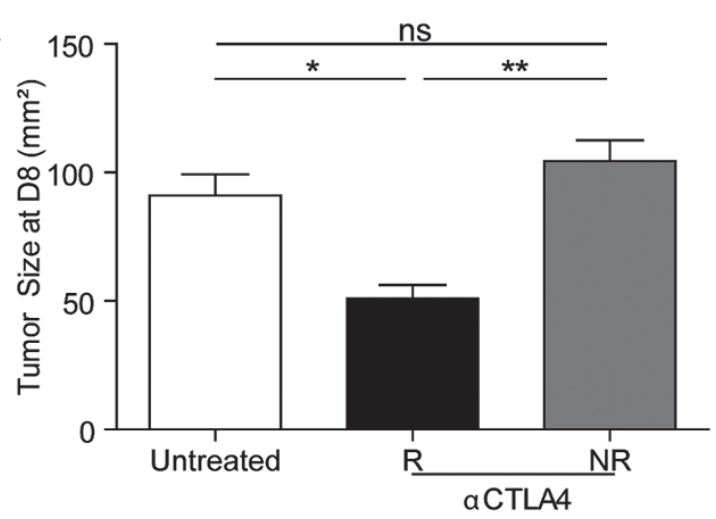

B

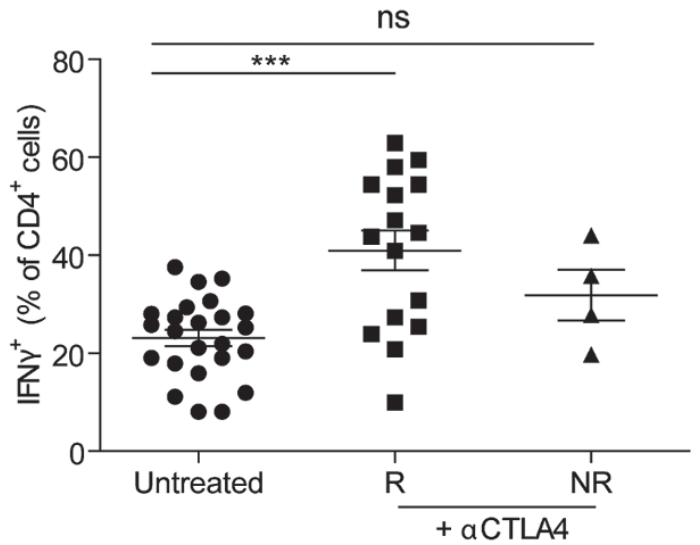

C

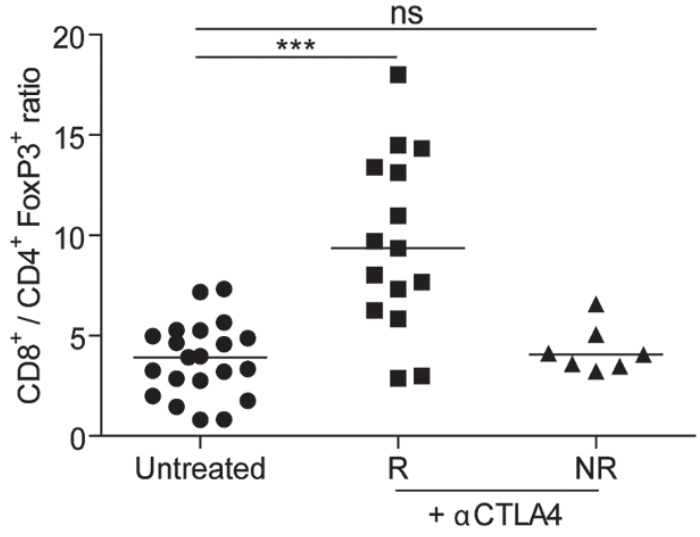

D

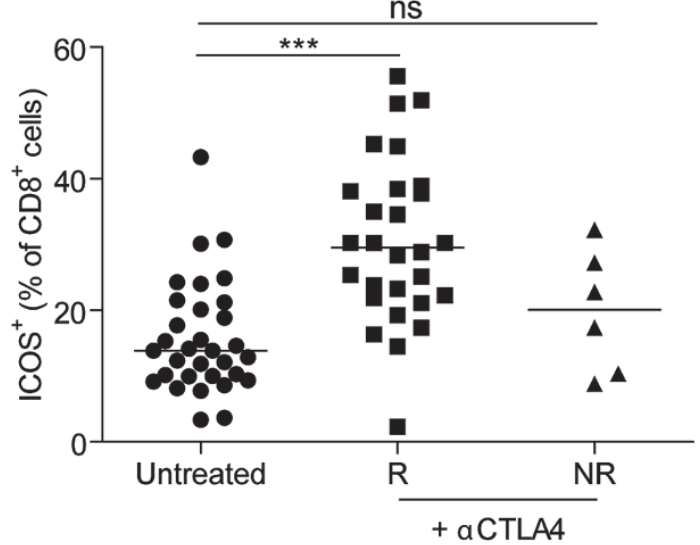

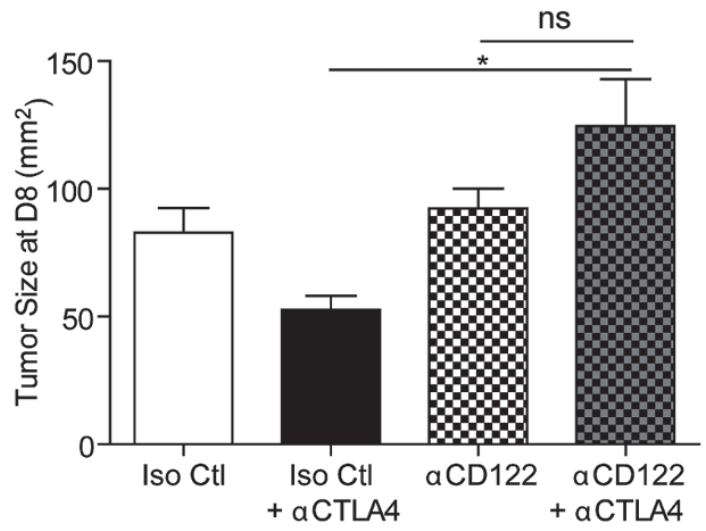
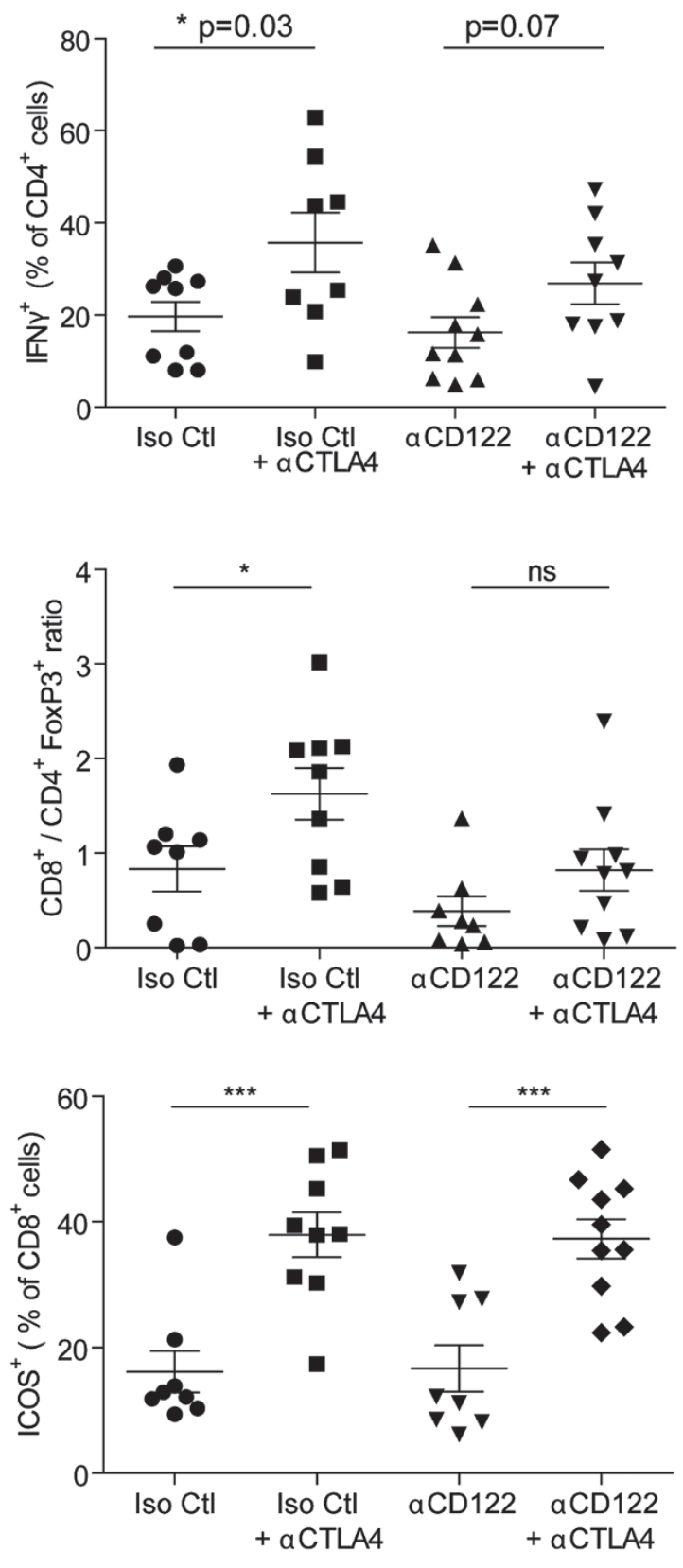
proportion of MM patients presented high baseline levels of sCD25 (above the median of normal volunteers: 330$1650 \mathrm{pg} / \mathrm{ml}$ [38]). Soluble CD25 reportedly behaves as a decoy receptor or mediates immunosuppressive effects mainly via Tregs $[30,31]$. Indeed, baseline concentrations of sCD25 in MM patients positively correlated with high circulating Treg numbers in a group of 27 patients whose peripheral blood mononucleated cells (PBMCs) were available [39] (Figure 4F).

As serum SCD25 concentrations did not increase after mCTLA4 blockade in tumor-bearing mice (Supplementary information, Figure S3), we investigated the functional impact of artificially raising serum sCD25 concentrations through iterative systemic infusions of high doses of recombinant sCD25 (Figure 5A, left panel). This manoeuver induced an expansion of $\mathrm{CD}^{+} \mathrm{Fox}-$ $\mathrm{P} 3{ }^{+} \mathrm{CD} 25^{\text {high }}$ Tregs in the blood on day 8 (Figure 5B), and in the spleen on day 15 (which was more evident in the presence of mCTLA-4 blockade; Figure 5C). External supply of sCD25 compromised the antitumor effects observed shortly after mCTLA-4 blockade (Figure 5D-5E). Moreover, sCD25 administration impaired anti-mCTLA-4 Ab-induced downregulation of FoxP3 expression on both Tregs $\left(\mathrm{CD} 4^{+} \mathrm{CD} 25^{\text {high }}\right)$ and $\mathrm{CD} 4^{+} \mathrm{Lag} 3^{+}$TILs (Figure $5 \mathrm{~F}$ ). In contrast, the elevated frequency of $\mathrm{CD} 8^{+}$ ICOS $^{+}$TILs induced by mCTLA-4 blockade (that was CD122-independent; Figure 2D, right panel) was not affected by sCD25 (data not shown).

Altogether, these preclinical data suggest that high circulating levels of sCD25 prior to therapy may curtail the antitumor effects of mCTLA-4 blockade.

Baseline sCD25 and lactate dehydrogenase (LDH) serum levels predict resistance to CTLA-4 blockade

The potential negative impact of a high baseline level of sCD25 on the clinical outcome of MM patients receiving ipilimumab was evaluated in a retrospective study encompassing 9 independent cohorts from 5 dif- ferent countries (Supplementary information, Table S1). An elevated serum level of LDH is known to be a poor prognostic factor in a number of cancers including melanoma, perhaps reflecting the anaerobic glycolysis of rapidly growing hypoxic tumors. The LDH parameter has been included in the 2009 AJCC staging system for melanoma, which subdivides MM into M1a (skin, lymph node or subcutaneous metastases only), M1b (lung metastases only) and M1c (visceral metastases or metastases at any site with a raised LDH serum level) [40]. Circulating pretreatment levels of sCD25 and LDH, immune response-related criteria (irRC) at 4 weeks and tumor staging (M1a, M1b, M1c) were all statistically associated with the overall survival (OS) in univariate analyses (Figure 6A-6B and Supplementary information, Table S1, Figures S4 and S5A, with S5A focusing on distinct cohorts). In contrast, neither sLAG3 levels nor absolute lymphocyte counts at diagnosis correlated with the OS (Supplementary information, Figure S5B and data not shown). The prognostic value of sCD25 remained significant (hazard ratio $(\mathrm{HR})=1.26$ [1.04; 1.54], $P<0.0165$ ) after including the LDH value in a multivariate model, suggesting an independent contribution of $\mathrm{sCD} 25$ to OS (Figure 6C). High sCD25 concentrations at diagnosis correlated with poor outcome, specifically in the subset with high LDH levels (Figure 6C). Similarly, high sCD25 levels observed 3 weeks (T21) post-ipilimumab treatment negatively predicted OS (Figure 6D), while the percentage raise of $\mathrm{sCD} 25$ at $\mathrm{T} 21$ with respect to $\mathrm{T} 0$ failed to do so (Supplementary information, Figure S5C). We conclude that, combined with LDH values, circulating $\mathrm{SCD} 25$ levels represent a valuable predictor of resistance to CTLA-4 blockade.

\section{Discussion}

Biomarkers that predict the long-term effects of expensive and potentially toxic treatments with im-

Figure 2 IL-2Rß-dependent TIL accumulation and elevated Teff/Treg ratio during mCTLA4 blockade. Heterogeneity of the antitumor responses obtained with mCTLA4 blockade. (A) Tumor sizes after 3 inoculations of anti-mCTLA-4 Ab and/or anti-CD122 Ab in responders (R) versus non responders (NR) in MCA-205-OVA tumor-bearers at time of sacrifice (10 mice/ group). (B) IL-2R $\beta$-dependent Th1 accumulation in tumor beds post-anti-mCTLA-4 Ab treatment. Flow cytometric analyses to determine the percentages of $\mathrm{CD} 45^{+} \mathrm{CD} 4^{+} \mathrm{T}$ cells among viable cells after enzymatic dissociation of MCA205-OVA tumors. CD4 ${ }^{+}$TILs were also subjected to the intracellular detection of IFN $\gamma$. Graphs depict the percentages of IFN $\gamma^{+}$cells in the gate of live $\mathrm{CD} 45^{+} \mathrm{CD} 3^{+} \mathrm{CD} 4^{+}$cells in $\mathrm{R}$ and NR mice after PMA-ionomycin stimulation. Each dot represents one tumor (left panel) and treated or not with anti-CD122 antibodies (right panel). (C) IL-2R $\beta$-dependent CD8 ${ }^{+}$Teff/Treg ratio in tumors post-anti-mCTLA-4 Ab treatment. Idem as in B, but reporting the percentage of $C D 8^{+}$cells in the gate of live $C D 45^{+} \mathrm{CD} 3^{+}$cells as well as that of $\mathrm{FoxP}^{+}$in the gate of live $\mathrm{CD} 45^{+} \mathrm{CD} 3^{+} \mathrm{CD} 4^{+}$cells, followed by calculation of the ratio between these two values for each tumor presented as one dot. (D) CD122-independent upregulation of ICOS expression on CD8 ${ }^{+}$T cells in tumors. Flow cytometric determination of ICOS expression gating on $\mathrm{CD} 45^{+} \mathrm{CD} 3^{+} \mathrm{CD} 8^{+}$live tumor-infiltrating cells. Results are presented as concatanated data from individual mice or tumors from several independent experiments. ${ }^{*} P<0.05,{ }^{* *} P<0.01$, ${ }^{* *} P<0.001$, ns, not significant (Student's $t$-test or ANOVA). 

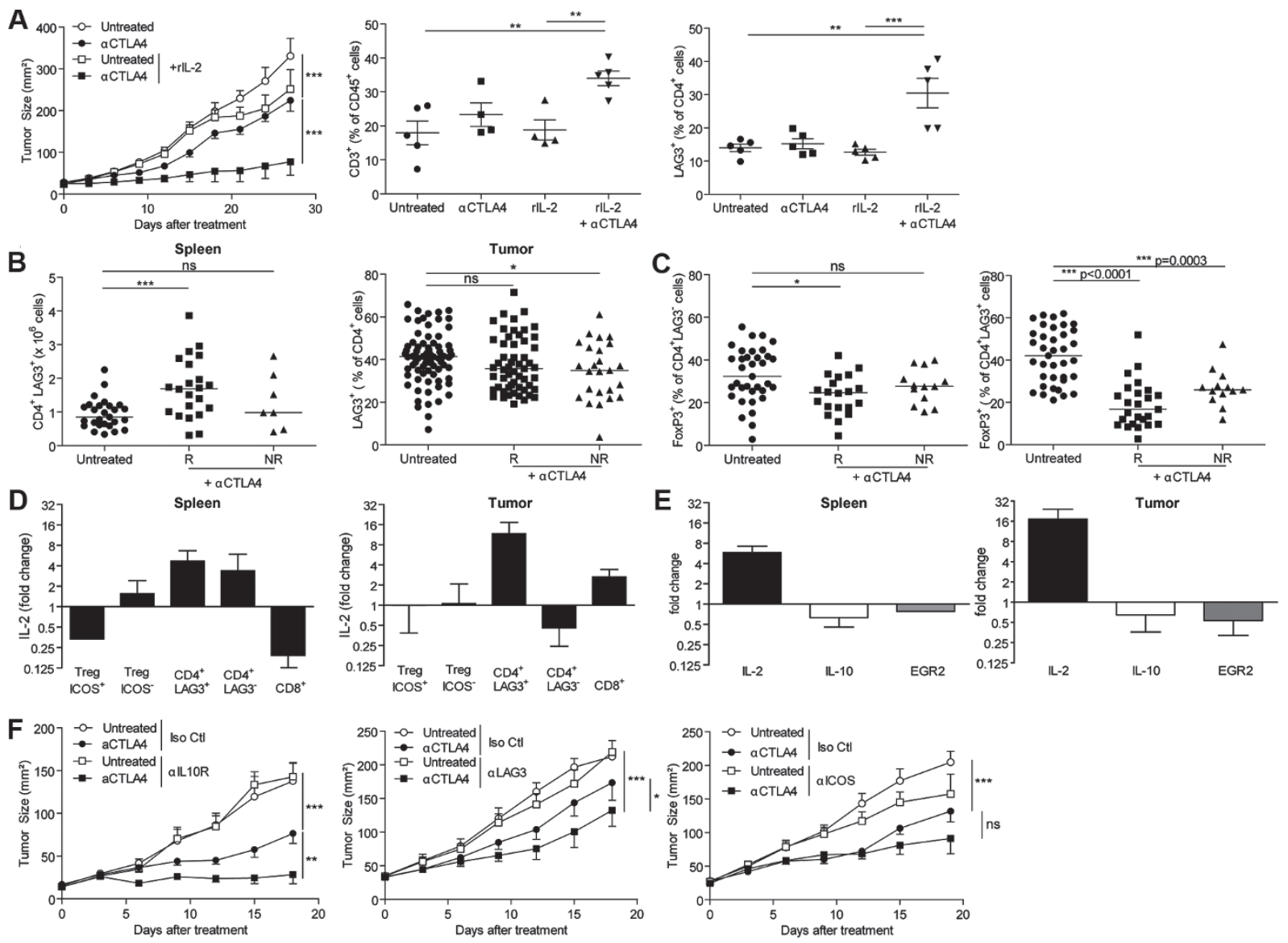

Figure $3 \mathrm{CD}^{+} \mathrm{Lag}^{+} \mathrm{T}$ cells produce IL-2 and lose FoxP3 expression in responders during anti-mCTLA-4 therapy. (A) rIL-2 synergizes with anti-mCTLA-4 Ab. Combination of rlL-2 and CTLA-4 blockade (left panel) led to enhanced tumor-suppressive effects. Flow cytometric analyses of $\mathrm{CD}^{+}$cells gating on live CD45 ${ }^{+}$blood cells on day 28 (middle panel) or CD4 ${ }^{+} \mathrm{Lag} 3^{+} \mathrm{T}$ cells in tumor beds (right panel) upon treatment with a combination of rIL-2 and anti-mCTLA-4 Ab. Each dot represents one tumor. Tumor growth kinetics (left panel) is depicted for one representative experiment (5-6 mice/group) out of 2-3 experiments yielding similar results. Tumor size is shown as mean \pm SEM. ${ }^{* *} P<0.01,{ }^{* * *} P<0.001$, ns, not significant (Student's $t$-test or ANOVA). (B) Lag3 expression on CD4 ${ }^{+} \mathrm{T}$ splenocytes and TILs. Flow cytometric analysis reveals Lag3 expression gating on $\mathrm{CD}^{+} \mathrm{CD} 4^{+} \mathrm{T}$ cells from the spleen (left panel) and tumors (right panel) from $\mathrm{R}$ and NR mice receiving anti-mCTLA-4 $\mathrm{Ab}$ and from untreated mice. Graphs represent concatenated data from three experiments. (C) Loss of FoxP3 expression on $\mathrm{CD}^{+}$Lag3 $^{-}$(left panel) or $\mathrm{CD}^{+} \mathrm{Lag3}^{+}$(right panel) TILs during mCTLA4 blockade. Flow cytometric determination of FoxP3 expression gating on $\mathrm{Lag}^{+}$versus $\mathrm{Lag}^{-} \mathrm{CD} 4^{+}$TILs in R and NR mice. Concatenated data from several independent experiments are shown. (D, E) CD4 ${ }^{+} \mathrm{Lag}^{+}$cells are the main IL-2 producers in tumors post-anti-mCTLA-4 treatment. Cell sorting of various $T$ cell subsets in spleens (D, left panel) or tumors (D, right panel) to determine IL-2 transcription by qRT-PCR. (E) Cell sorting of $\mathrm{CD}^{+} \mathrm{Lag}^{+} \mathrm{T}$ cells to determine gene transcription by qRT-PCR. Ratio of transcription levels of $I L-2, I L-10$ and Egr2 in the $\mathrm{CD}^{+}{ }^{+} \mathrm{Lag}^{+} \mathrm{T}$ cell subset from spleens (E, left panel) or tumors (E, right panel) of untreated versus anti-mCTLA-4 Ab-treated mice on day 8. Results are presented as mean ratios of tumors from several independent experiments. (F) Effects of neutralizing IL-10R (left panel), Lag3 (middle panel) or ICOS (right panel) on tumor growth following mCTLA-4 blockade. Anti-ICOS and anti-LAG3 neutralizing Abs were injected i.p. 1 day before each anti-CTLA-4 treatment. IL-10R-specific neutralizing $\mathrm{Ab}$ was injected daily. Results are presented as mean ratios from several independent experiments. ${ }^{\star} P<0.05$, ${ }^{\star *} P<$ $0.01,{ }^{* * *} P<0.001$, ns, not significant (Student's $t$-test or ANOVA).

mune-checkpoint blockers may be used to significantly improve the clinical and economical management of cancer patients. Here, we used a mechanism-centered approach that led to the discovery of a novel biomarker relevant to CTLA4 blockade in MM patients. In summa- ry, we found that (i) mCTLA-4 blockade improved the intratumoral Teff/Treg ratio and reduced tumor growth only in conditions in which either IL-2 or IL-15 could act on IL-2R $\beta$ chains; (ii) CTLA-4 blockade provoked a reduction of immunosuppressive $\mathrm{CD} 4{ }^{+} \mathrm{Lag} 33^{+} \mathrm{ICOS}^{+} \mathrm{IL}-10^{+}$ 
T lymphocytes with a concomitant rise in IL-2-producing cells; and (iii) external supply of sCD25 subverted the therapeutic effects of CTLA-4 blockade in mice, while high baseline serum levels of sCD25 were associated with compromised ipilimumab effects in MM patients.

The immunostimulatory action of mCTLA-4 blockade has been well studied $[13,41]$. CTLA-4 is a critical negative regulator of antitumor immunity [41] and an essential mediator of immune homeostasis as illuminated by the fatal lymphoproliferative disorder in CTLA-4-deficient mice [42]. A pioneering report unveiled the role of IL-2 in mediating the anti-CTLA-4 Ab-induced proliferation of adoptively transferred OT-1 T cells (which express an ovalbumin-specific TCR), as well as their accumulation in the peritoneal cavity harboring OVA-expressing tumors. In this model system, CTLA-4 blockade stimulated IL-2-secreting $\mathrm{CD}^{+} \mathrm{T}$ helper cells, which were indispensable for the sustained effector (lytic) function of $\mathrm{CD} 8^{+} \mathrm{CD} 25^{+} \mathrm{T}$ cells [28]. Anti-CTLA-4-IgG2a and to a lesser extent anti-CTLA-4-IgG2b may cause the elimination of Tregs from the tumor site, consistent with their ability to bind to Fc $\gamma$ Rs [12]. This was accompanied by the activation and expansion of effector T cells, which might either result directly from alleviating inhibitory CTLA-4-B7 interactions or indirectly from Treg depletion. The functional relevance of the balance between these two antagonistic $\mathrm{T}$ cell subsets has been previously highlighted in mice, in the context of GVAX-based vaccines and CTLA-4 blockade against B16 melanomas, as well as in advanced ovarian carcinoma patients [18, 43]. Our data revealed that two responses that were strongly associated with tumor regression after mCTLA-4 blockade, namely the depletions of Fox $3^{+}$Treg and $\mathrm{CD} 4^{+} \mathrm{Lag} 3^{+} \mathrm{FoxP}^{+}{ }^{+}$TILs, were compromised by sCD 25 treatment (Figure 5F). High doses of anti-CD25 depleting $\mathrm{Ab}$ that decreased circulating and intratumoral effector $\mathrm{T}$ cells [28] (data not shown) also compromised the therapeutic efficacy of CTLA-4 blockade (Figure 1D). Neutralizing the IL-2R $\beta$ chain CD122 similarly abolished the rise in the Teff/Treg ratio that normally follows mCTLA-4 blockade (Figure 2C). Altogether, our findings support the notion that the IL-2/IL-2R $\alpha \beta$ signaling pathway is indispensable for facilitating the stimulation of $\mathrm{CD} 4^{+} \mathrm{T}$ helper and $\mathrm{CD} 8^{+} \mathrm{T}$ effector cells in the context of mCTLA-4 blockade.

Our results also revealed that the principal source of intratumoral IL-2 after mCTLA-4 blockade was a CD4 ${ }^{+}$ $\mathrm{T}$ cell subset expressing Lag3. Lag3 co-engagement during TCR/CD3 ligation mediates T cell suppression $[36,44]$. In accord with previous reports on intestinal $\mathrm{CD} 4^{+} \mathrm{Lag}_{3}{ }^{+}$cells [36], the $\mathrm{CD} 4^{+} \mathrm{Lag} 3^{+}$subset identified here also expressed high levels of IL-10 within tumors (data not shown). Roncarollo's group recently identified a population of IL-10-producing $\operatorname{Tr} 1$ cells (the CD$49 \mathrm{~b}^{+}$Lag $3^{+}$subset) exhibiting a strong immunosuppressive phenotype, which express high levels of CTLA-4 [45] and hence might be targeted by CTLA-4 blockade. A portion of the $\mathrm{CD} 4^{+} \mathrm{Lag} 3^{+}$subset identified here constitutively expressed ICOS, yet downregulated ICOS upon mCTLA-4 blockade, especially in responders (data not shown). Similarly, a fraction of intratumoral $\mathrm{CD} 4^{+} \mathrm{Lag} 3^{+}$ $\mathrm{T}$ cells expressed FoxP3 in control conditions, yet exhibiting downregulated FoxP3 expression upon mCTLA-4 blockade (Figure 3C). Furthermore, this intratumoral $\mathrm{CD} 4^{+} \mathrm{Lag} 3^{+}$TIL subset also expressed Egr2 before treatment (data not shown), likely indicating a state of anergy coupled to tumor progression. Conditional deletion of Egr2 in peripheral T cells reportedly restored Ras/MAPK signaling, IL-2 secretion and proliferation [46], while overexpression of Egr2 in $\mathrm{CD}^{+} \mathrm{T}$ lymphocytes resulted in the generation of immunosuppressive $\mathrm{CD} 49 \mathrm{~b}^{+} \mathrm{Lag} 3^{+}$ cells [36]. The additive antitumor effects resulting from simultaneous blockade of CTLA-4 and IL-10R or that of CTLA4 and Lag3 (Figure 3F) support the idea that CD$49 \mathrm{~b}^{+}$Lag $3^{+}$TILs are immunosuppressive. While CTLA4 blockade positively regulated $I L-2$ transcription, it caused a merely partial downregulation of $I L-10$ and Egr2 transcription in tumor beds, which may explain why the simultaneous neutralization of other immune checkpoints (including that conferred by IL-10R) could further improve the therapeutic efficacy of CTLA-4 blockade (Figure 3F).

In addition to membrane-anchored IL-2R $\alpha(55 \mathrm{kDa})$, a truncated soluble form of the CD25 receptor (sIL-2R $\alpha$ / $\mathrm{sCD} 25)$ (45 kDa) efficiently binds to IL-2. sCD25 is generated as a monomeric protein from the membrane-bound form through proteolytic cleavage or 'shedding', and its serum concentration is directly related to the proliferation rate of activated T cells [38]. Elevated serum sCD25 levels have been detected in autoimmune and inflammatory diseases, as well as in cancer. In tumor-bearers, IL$2 \mathrm{R} \alpha$ is expressed on activated immune cells (lymphocytes or monocytes) and, at least in some cases, non-stimulated malignant cells. Intravenous infusion of high doses of rIL-2 for T cell activation also induces an elevation in serum sCD25 levels [47]. Similarly, our current study revealed that low doses of rIL-2 that reportedly caused Treg expansion and suppressed cryoglobulinemia-related vasculitis in patients with hepatitis C [37] elicited an increase in $\mathrm{sCD} 25$ serum levels comparable to that observed in patients treated with one infusion of ipilimumab (Figure 4A-4B). Intriguingly, low doses of ipilimumab $(<3 \mathrm{mg} / \mathrm{kg}$, in conjunction with rGM-CSF) could promote an increase in the frequency of circulating Tregs 
A

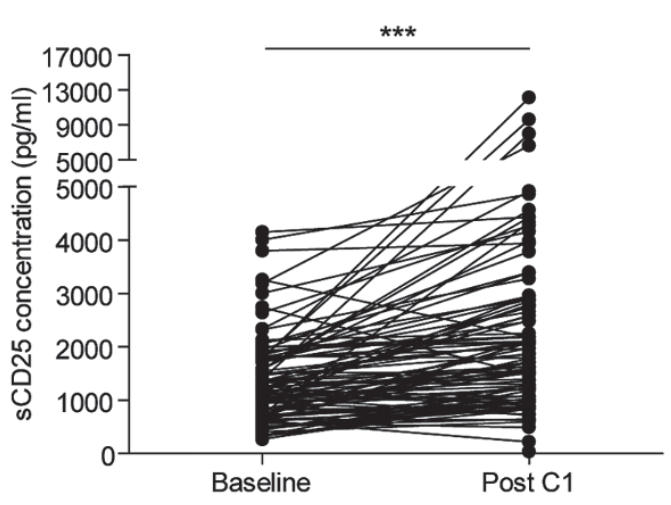

C

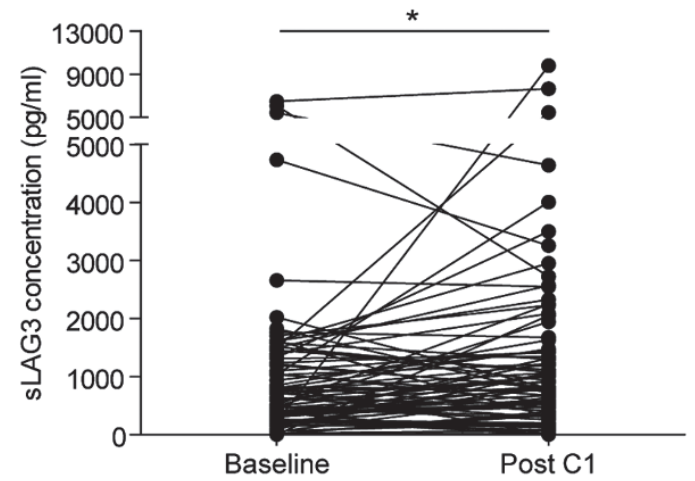

E

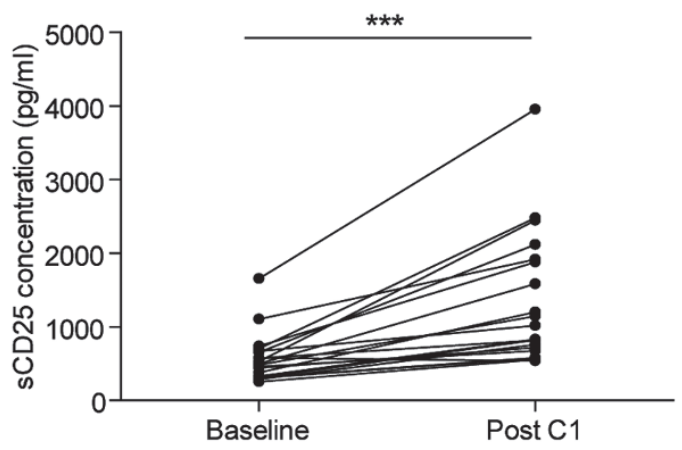

B

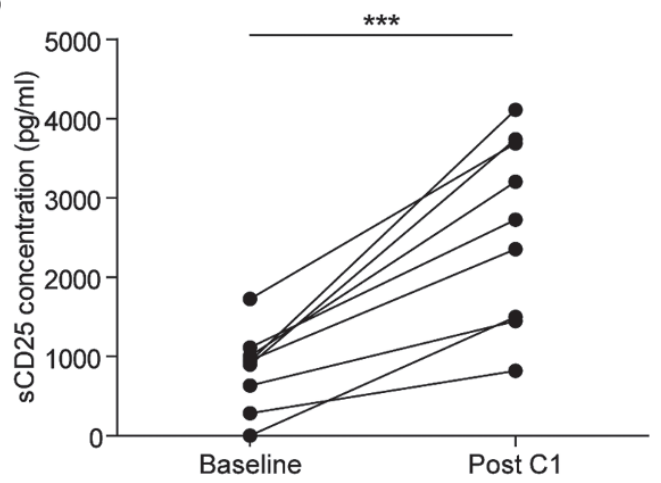

D

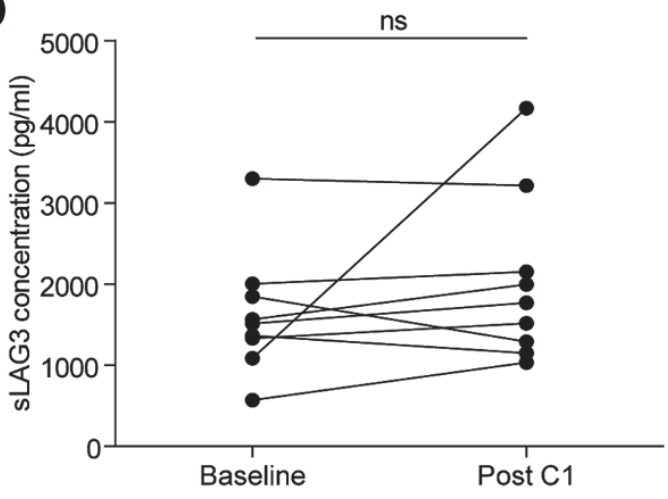

$\mathbf{F}$

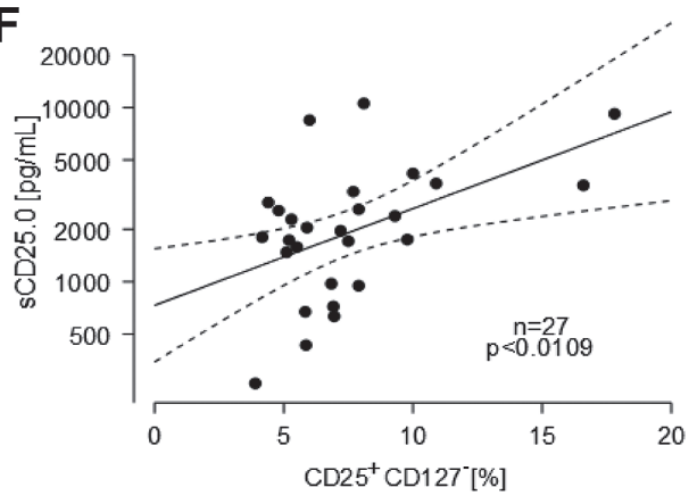

Figure 4 Serum levels of SCD25 in MM patients. (A-D) Serum levels of SCD25 and sLag3 in patients. Ninety-nine MM patients treated with ipilimumab were analyzed and compared with one cohort of 9 patients with an autoimmune disease treated with low-dose rlL-2. Graphs depict the serum concentrations of SCD25 (A-B) or SLag3 (C-D) prior to and 3 weeks after ipilimumab (A, C) or rlL-2 (B, D) treatment. Each dot represents one patient. ${ }^{*} P<0.05$, ${ }^{* * *} P<0.001$, ns, not significant (Paired Student's t-test or ANOVA). (E) Effects of tremelimumab on serum SCD25 levels. A cohort of $20 \mathrm{MM}$ patients received $15 \mathrm{mg} /$ $\mathrm{kg}$ of tremelimumab. Serum SCD25 levels were measured before and 3 weeks after the first injection. ${ }^{* * *} P<0.001$ (Paired Student's $t$-test). (F) Correlations between serum SCD25 levels and circulating Treg percentages. SCD25 levels and percentages of $\mathrm{CD} 4^{+} \mathrm{CD} 25^{\text {high }} \mathrm{CD} 127^{-}$Tregs of $27 \mathrm{MM}$ patients were determined at diagnosis before ipilimumab therapy.

[39]. Based on these premises, it is tempting to speculate that high sCD25 serum concentrations could reflect an
IL-2-mediated Treg proliferation, thus explaining the positive correlation between $\mathrm{SCD} 25$ levels and circulat- 
ing Tregs in MM patients (Figure 4F).

The clinical relevance of serum sCD25 has been evaluated in adults and infants [38]. Highly supranormal sCD25 levels are often found in lymphoproliferative disorders. In contrast, solid malignancies usually are not associated with elevations in serum sCD25 unless the disease has disseminated [48-51]. Elevated sCD25 levels in the ascites of ovarian cancer patients and in the serum of breast cancer patients correlated with a low frequency of TILs, suggesting that SCD25 could inhibit antitumor immunosurveillance [52]. sCD25 suppresses IL-2-induced surface $\mathrm{CD} 25$ expression and $\mathrm{T}$ cell responses in vitro [30, 31, 53-55]. Russell et al. [56] reported that $\mathrm{SCD} 25$ can exacerbate experimental autoimmune encephalitis by acting as a decoy receptor that sequesters IL-2, eventually altering the balance between Th1/Th17 and Tregs. A different mechanism might explain the capacity of sCD25 to subvert the immunostimulatory effects of CTLA-4 blockade. Both preclinical (Figure 5) and clinical data (Figure 6A-6B) indicate that the tumor growth-restraining effect of CTLA-4 blockade is compromised when the circulating sCD25 concentration is high, paralleling elevated Treg proportions. In our study, it is conceivable that $\mathrm{sCD} 25$ directly binds to IL-2, causing a preferential Treg amplification. In MM patients with LDH levels of $>500 \mathrm{IU} / \mathrm{ml}$, the elevated $\mathrm{sCD} 25$ serum concentration had a negative prognostic impact, allowing the identification of a subset of $\sim 25 \%$ patients that did not respond to ipilimumab (Figure 6C). Our data that high LDH levels $(>250 \mathrm{IU} / \mathrm{ml})$ correlated with poor clinical outcome of ipilimumab-treated MM are in accordance with the two phase III trials leading to FDA and EMA approval of ipilimumab. Indeed, low LDH serum levels were associated with an increased OS $[4,5]$. In smaller cohorts treated with ipilimumab reported by Wilgenhof et al. [57] and Delyon et al. [17], the elevated LDH level was also negatively associated with survival. Moreover, a recent report analyzing several prognostic parameters in two European MM cohorts treated with $3 \mathrm{mg} / \mathrm{kg}$ of ipilimumab concluded that an LDH level of $>2$ folds above the normal range correlated with clinical failure of CTLA4 blockade [58]. Nevertheless these retrospective results must be confirmed in prospective studies before we can test whether MM patients with high $\mathrm{sCD} 25$ and LDH serum concentrations should receive agents targeting CD25 such as radioimmunoconjugates or immunotoxins [59]. Altogether, we anticipate that the critical role of IL-2/ IL-2R signaling in the regulation of ipilimumab action would have major clinical implications.

\section{Materials and Methods}

\section{Patients and specimens}

Patient samples (plasma or sera) were provided by the Gustave Roussy Institute (Villejuif, France), the Memorial Sloan-Kettering Cancer Center (New York, USA), the Siena University Hospital (Siena, Italy), the German Cancer Research Center (Heidelberg, Germany), the Poznan University of Medical Sciences (Poznan, Poland) and the Essen University Hospital (Essen, Germany). Clinical responses were classified according to the Response Evaluation Criteria in Solid Tumors (RECIST) criteria and the immune-related response criteria (irRC) (Supplementary information, Table S1). The immunomonitoring studies on fresh PBMCs were prospectively conducted on 27 patients.

\section{Serum levels of $S C D 25$ and $s L A G 3$}

Serum sCD25 and sLAG3 concentrations were measured using the sIL-2 Receptor EIA Kit (Beckman Coulter, Miami, FL, USA) and the sLAG3 Duo set (R\&D systems, Minneapolis, MN, USA). Patient sera (or plasma) were collected before treatment and after the first and the second ipilimumab administrations.

\section{Quantitative reverse-transcription $P C R$}

$\mathrm{CD}^{+} \mathrm{LAG}^{+}, \mathrm{CD}^{+} \mathrm{LAG}^{-}, \mathrm{CD} 127^{\text {low }} \mathrm{CD} 25^{+} \mathrm{ICOS}^{+}, \mathrm{CD} 127^{\text {low }-}$ $\mathrm{CD}_{25}{ }^{+} \mathrm{ICOS}^{-}$and $\mathrm{CD} 8^{+} \mathrm{T}$-cell populations were sorted by flow cytometry (BD Influx and BD ARIA III with FACSDiva software) from murine spleens and tumors and placed in RLT buffer (Qiagen, Hilden, Germany) containing 1\% 2-mercaptoethanol, followed by their storage at $-80{ }^{\circ} \mathrm{C}$. Total cellular RNA was isolated with the RNeasy kit from Qiagen. All samples were treated with DNase using the RNase-free DNase Set (Qiagen) during the total RNA isolation. First-strand complementary DNA (cDNA) was synthesized from $5 \mu \mathrm{g}$ of total RNA with SuperScript III reverse transcriptase and random primers (Promega, Madison, WI, USA). Expression of $i l-2, i l-10$ and $e g r-2$ genes was analyzed by means of TaqMan Gene Expression Assays using the Universal Master Mix II (with UNG) (both from Applied Biosystems, Foster City, CA, USA), on a StepOnePlus Real-Time PCR System (Applied Biosystems). All Taqman primers were purchased from Life Technologies using FAM-TAMRA probes. Assay IDs were as follows: IL-2: Mm00434256_m1; IL-10: Mm00439614_m1; Egr2: Mm00456650 m1; PPIA: Mm03302254 g1. Quantitative RTPCR data were invariably normalized to the expression levels of the housekeeping gene peptidylprolyl isomerase A (Ppia). Quantification of fold changes was calculated using untreated samples as the control.

\section{Mice}

Mice aged between 7 and 12 weeks were used. Female C57BL/6J mice were obtained from Harlan (Gannat, France). Ifnarl $1^{-/}$C57BL/6J mice were kindly provided by Gilles Uzé (UMR 5235, Montpellier Fance). Mice were maintained in specific pathogen-free conditions at the Institut Gustave Roussy (IGR, Villejuif, France), the Peter MacCallum Cancer Center (East Melbourne, Australia) or the QIMR Berghofer Medical Research Institute and housed in a temperature-controlled environment with $12 \mathrm{~h}$ lightdark cycle and received food and water ad libitum. Experiments were carried out in compliance with French and European laws and regulations of the Peter MacCallum and QIMR Berghofer Animal Experimentation Ethics Committees. 
A

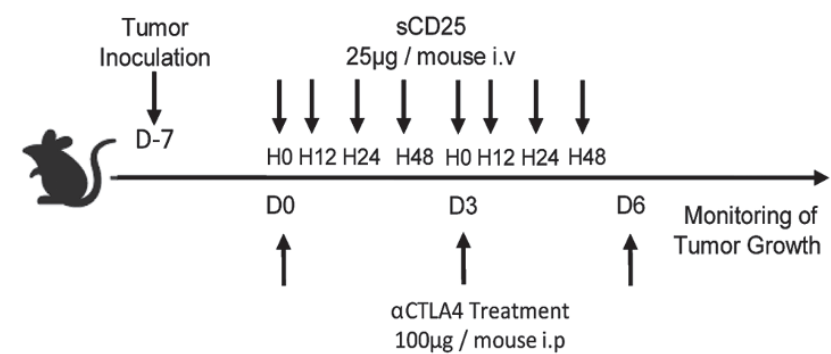

C

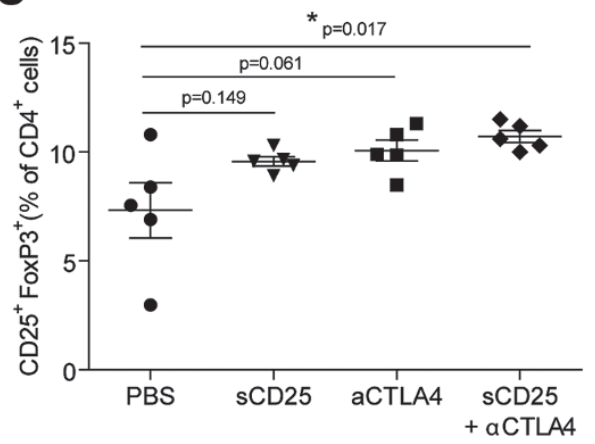

D

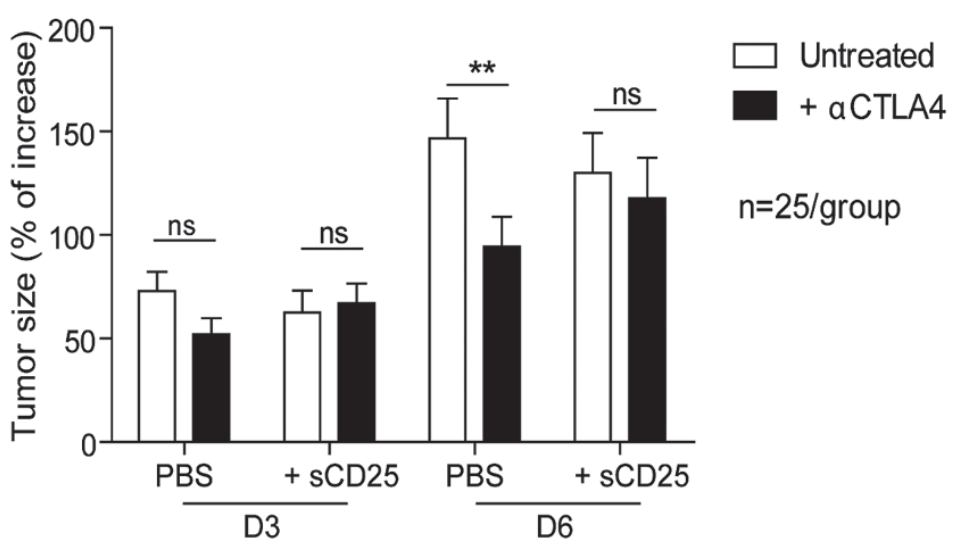

F

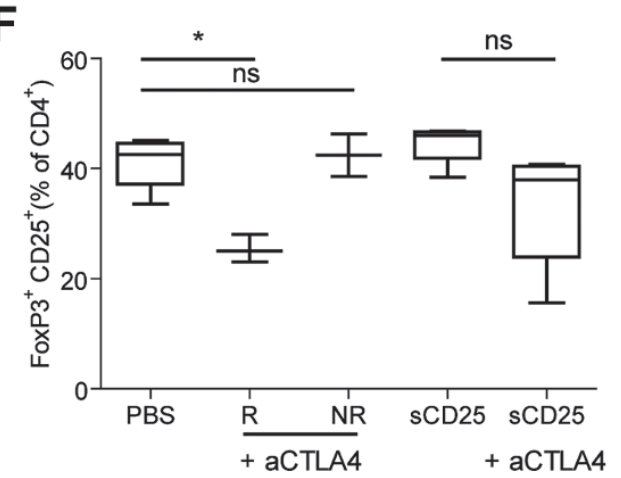

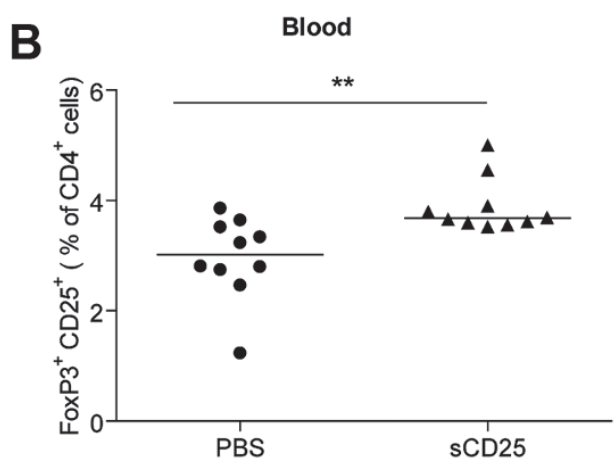

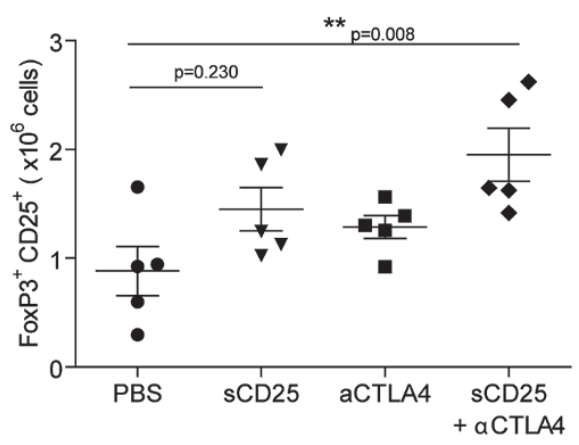

E

Day 0
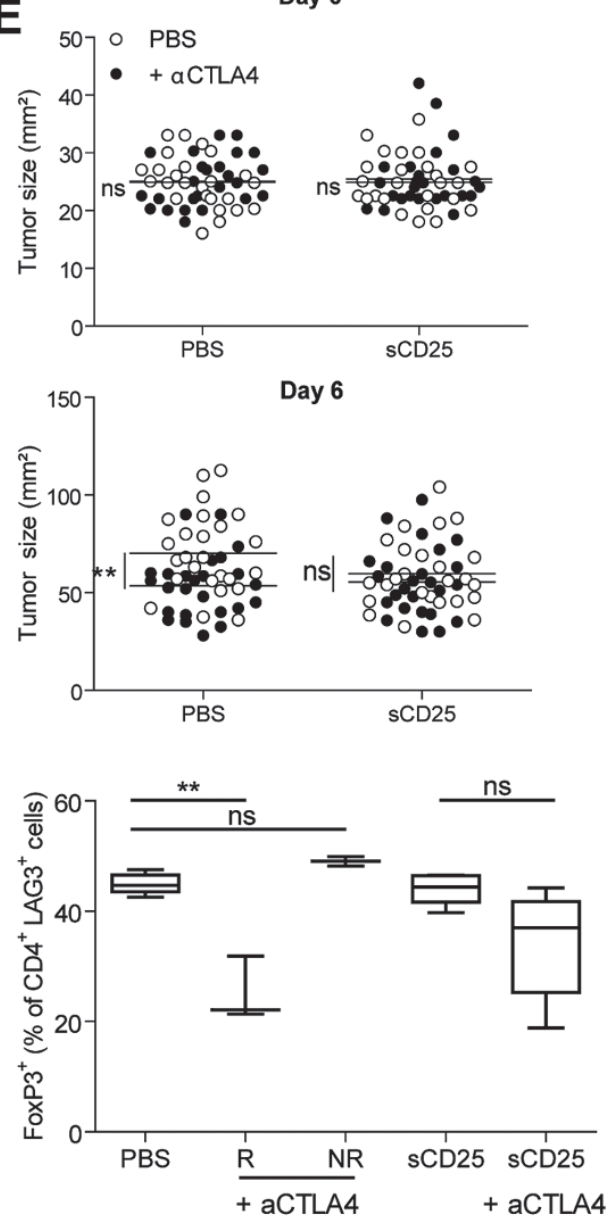


\section{Cell culture and reagents}

OVA-expressing mouse fibrosarcoma MCA205 cells (class I MHC H-2 ${ }^{\mathrm{b}}$, syngenic for C57BL/6 mice) were cultured at $37{ }^{\circ} \mathrm{C}$ under $5 \% \mathrm{CO}_{2}$ in RPMI-1640 medium supplemented with $10 \%$ heat-inactivated fetal bovine serum, 100 units $/ \mathrm{mL}$ penicillin $\mathrm{G}$ sodium, $100 \mu \mathrm{g} / \mathrm{mL}$ streptomycin sulfate, $2 \mathrm{mM}$ L-glutamine and $1 \mathrm{mM}$ sodium pyruvate and non-essential amino acids (Gibco-Invitrogen, Carlsbad, CA, USA). OVA-expressing MCA205 cells selected in complete medium (as above) further supplemented with $50 \mu \mathrm{g} / \mathrm{mL}$ hygromycin B (Invitrogen, Life Technologies). Murine colon carcinoma MC38 OVA $^{\text {dim }}$ cells were cultured in complete DMEM medium.

\section{Tumor challenge and treatment}

Mice were subcutaneously injected in the right flank with $1 \times$ $10^{6}$ MCA205-OVA (clone 9D9 kindly supplied by James Allison, University of Texas, MD Anderson, Houston, TX, USA) or $1 \times 10^{5}$

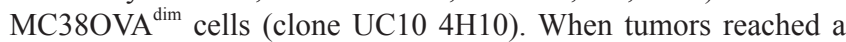
size of $25-30 \mathrm{~mm}^{2}$, mice were injected intraperitoneally (i.p) either with $100 \mu \mathrm{g}$ of anti-CTLA-4 (clone 9D9) [60] or with $100 \mu \mathrm{g}$ of anti-programmed cell death protein 1 (clone RMP1-14) from BioXcell (West Lebanon, NH, USA). Mice were injected 5 times at 3-day intervals with 9D9 or 4 times at 4 days intervals for RMP114 , and tumor size was routinely monitored by means of a caliper.

Anti-CD122 (clone TM-beta1), anti-CD25 (clone PC-61.5.3), anti-IL10R (clone 1B1.3A), anti-LAG3 (clone C9B7W), anti-ICOS (clone 17G9), and rat IgG (LTF-2, HRPN) used in vivo were obtained from BioXcell, and anti-IL-2 (JES6-1A12) and anti-IL-15 (AIO.3) were provided by eBioscience (San Diego, CA, USA). Mice received $200 \mu \mathrm{g}$ of anti-CD122, $250 \mu \mathrm{g}$ of anti-CD25, $500 \mu \mathrm{g}$ of anti-IL-10R, $200 \mu \mathrm{g}$ of anti-LAG3 and $250 \mu \mathrm{g}$ of anti-ICOS, injected i.p. 1 day before each anti-mCTLA-4 injection. Anti-IL-15 and anti-IL-2 were administered into the tumor bed at $20 \mu \mathrm{g}$ on day 0 and day 3 and $10 \mu \mathrm{g}$ per mouse on day 6 after CTLA-4 blockade. sCD25 (recombinant human IL-2R $\alpha$, Peprotech, Rocky Hill, NJ, USA) was injected i.v. at $25 \mu \mathrm{g}$ ).

\section{In vivo depletion of $\mathrm{T}$ or $\mathrm{NK}$ cells}

Mice were injected i.p. with $100 \mu \mathrm{g}$ of either rat anti-mouse CD8 (clone 53-6.72) or rat anti-mouse CD4 (clone GK 1.5) 1 day before anti-CTLA-4 injection, and every 3 days during the experiment. In vivo depletion of NK cells was accomplished using
anti-NK1.1 (clone PK146) at $150 \mu \mathrm{g}$ per mouse, administered i.p. daily for 3 days before anti-CTLA-4 injection and every 2-3 days throughout the experiment. Isotype control antibodies including rat IgG (clone LTF-2 or 2A3) and mouse IgG (clone C1.18.4) were given at the same dosages as controls. All the antibodies were purchased from BioXcell.

\section{Flow cytometry}

Eight color flow cytometric analysis was performed with antibodies conjugated to fluorescein isothiocyanate, phycoerythrin, phycoerythrin cyanin 7 , peridinin chlorophyll protein cyanin 5.5 , allophycocyanin cyanin 7 , pacific blue or allophycocyanin. The staining was realized on splenocytes or monocellular suspensions from mouse tumors. Tumors were obtained 2 days after the third injection of anti-CTLA-4. Excised tumors were cut into small pieces and digested in RPMI medium containing Liberase at $25 \mu \mathrm{g} / \mathrm{mL}$ (Roche) and DNase 1 at $150 \mathrm{UI} / \mathrm{mL}$ (Roche) for $30 \mathrm{~min}$ at $37^{\circ} \mathrm{C}$, followed by crushing the pieces on a $100 \mu \mathrm{m}$ cell strainer. Two millions of splenocytes (after red blood cells lysis) or tumor cells were preincubated with purified anti-mouse CD16/CD32 (clone 93 purchased from eBioscience) for $15 \mathrm{~min}$ at $4{ }^{\circ} \mathrm{C}$, before the membrane staining. For intracellular staining, the FoxP3 staining kit (eBioscience) was used. Dead cells were excluded using the Live/Dead Fixable Yellow dead cell stain kit (Life Technologies). Stained samples were run on a Canto II (BD Bioscience, San Jose, CA, USA) cytometer, and analyses were performed with FlowJo software (Tree Star, Ashland, OR, USA). For cytokine staining, cells were stimulated for $4 \mathrm{~h}$ at $37{ }^{\circ} \mathrm{C}$ with $50 \mathrm{ng} / \mathrm{mL}$ of phorbol 12 -myristate 13 -acetate (PMA), 1 $\mu \mathrm{g} / \mathrm{mL}$ of ionomycin (Calbiochem) and BD Golgi STOP (BD Biosciences). Anti-CD45.2 (104), CD127 (A7R34), FoxP3 (FJK-16s), LAG3 (eBioC9B7W), ICOS (7E17G9), ICOSL (HK5.3), IL17a (eBio17B7), IFN- $\gamma$ (XMG1.2), TNF- $\alpha$ (MP6-XT22) and isotype controls rat IgG1 (eBRG1), IgG2a (eBRG2a), IgG2b (eBRG2b) were purchased from eBioscience. Anti-CD3 (145-2C11), CD25 (PC61.5.3), IL-10 (554467), CD11b (M1/70) and rat IgG1 were obtained from BD Bioscience. Anti-CD4 (GK1.5), CD8ß (YTS1567.7), $\mathrm{CD} 11 \mathrm{c}(\mathrm{N} 418)$ and rat IgG2a (RTK2758) were purchased from Biolegend (San Diego, CA, USA). Anti-CD25 (7D4) was from Miltenyi Biotech. For cytofluorometry of human PBMCs, anti-CD3 (BW262/56) and mouse IgG1 (IS5-21F5) were purchased from Miltenyi Biotech. Anti-CD4 (SK3), CD8 (SK1), CD25 (M-A251), CD45 (J33) and mouse IgG1 (MOPC-21) were obtained from BD Bioscience. Anti FoxP3 (PCH101) was purchased from eBioscience

Figure 5 sCD25 subverts the anticancer effects of anti-CTLA-4 blockade. (A) Experimental setting. sCD25 (0.2 mg in total) was administered systemically (i.v.) prior to and following the injections of anti-CTLA-4 Ab in MCA205-OVA tumor-bearers. (B) Monitoring of Tregs in the blood during administration of sCD25. The percentages of CD4 ${ }^{+} F o x P 3^{+} C D 25^{\text {high }} \mathrm{T}$ cells in the blood were determined on day 8 after the first sCD25 inoculation. (C) Monitoring Tregs in the spleen during administration of anti-mCTLA4 and/or sCD25. The percentages (left panel) or absolute numbers (right panel) of CD $4^{+}$FoxP $3^{+}$CD2 $25^{\text {high }} \mathrm{T}$ cells in the spleen were determined on day 15. (D, E) Inhibitory effects of sCD25 on the efficacy of mCTLA-4 blockade. Tumor sizes were monitored during the first 6 days of SCD25 administration at three time points (day 0 , day 3 and day 6 ). The increase in tumor size relative to baseline level (day 0 ) is depicted in $\mathbf{D}$, and tumor sizes of individual mice are indicated in E for anti-mCTLA-4 Ab-treated (black dots) or PBS-treated (empty dots) mice with or without sCD25 treatment. Each group comprised 5 or 10 mice. Three experiments were performed and concatenated. (F) Treatment with sCD25 reversed anti-CTLA4-induced FoxP3 depletion in TILs. The effects of SCD25 on the composition of TILs were examined in each group treated with anti-mCTLA-4 Ab alone (responding (R) or not (NR)) or in combination with SCD25. Flow cytometry was used to monitor the frequency of Tregs among live CD4 ${ }^{+}$TILs (left panel) and CD4 ${ }^{+}$Lag3 ${ }^{+}$TILs (right panel) in mice sacrificed on day 15. Note that none of the mice receiving the $\mathrm{sCD} 25^{+}$anti-mCTLA-4 combinational therapy responded on day 15 . Similar results were obtained on day 8 (data not shown). ${ }^{*} P<0.05$, ${ }^{* *} P<0.01$, ns, not significant (Paired Student's $t$-test or ANOVA). 
A
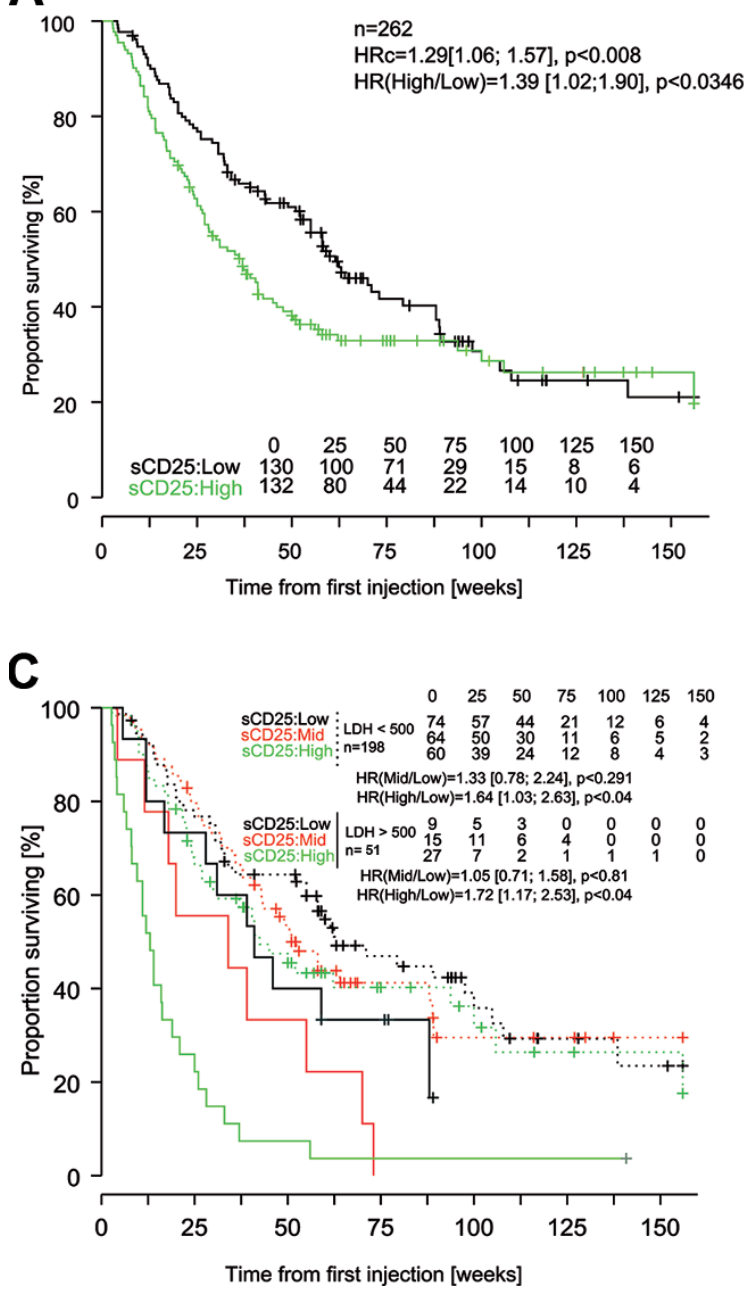

B

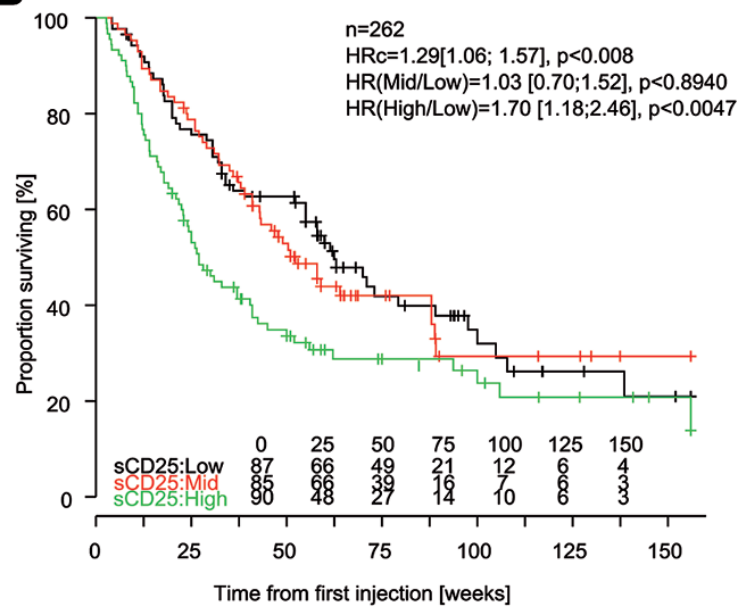

D

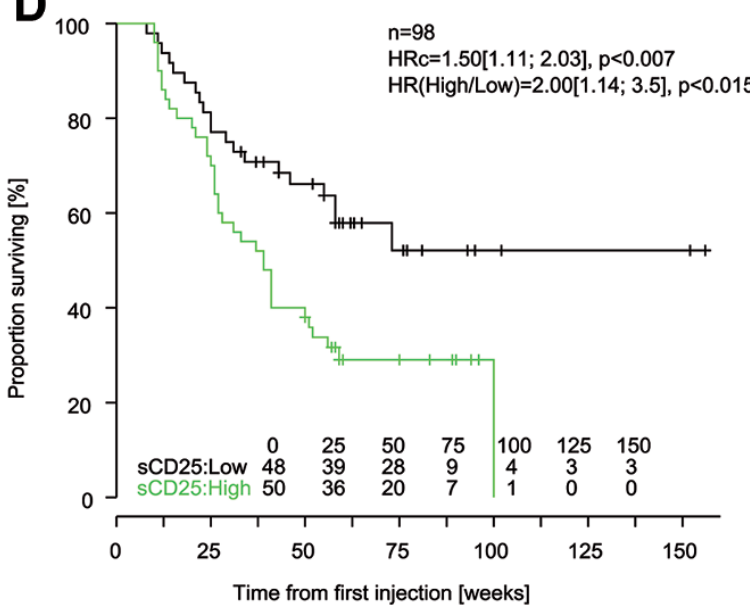

Figure 6 Baseline sCD25 serum levels predict resistance to ipilimumab in MM patients. (A, B) Baseline serum levels of sCD25 and overall survival (OS). Kaplan-Meier survival curves of all 262 patients (all treated with ipilimumab) segregated into two groups according to the median LRT $=4.69, P<0.030$ in A; tercile LRT $=9.17, P<0.010$ in $\mathbf{B}$. (C) Baseline serum levels of SCD25 and LDH, and OS. Kaplan-Meier curves of $262 \mathrm{MM}$ patients segregated into 6 groups according to the median value of $\mathrm{LDH}$ (with $500 \mathrm{IU} / \mathrm{ml}$ as a threshold) and the tercile of sCD25 LRT $=5.76, P=0.056$. (D) OS and serum sCD25 levels measured after ipilimumab injections. Kaplan-Meier curves of $98 \mathrm{MM}$ patients segregated into two groups according to the median value of SCD25 post C1 (3 weeks post-first ipilimumab injection). LRT $=6.01, P<0.014$.

and anti-LAG3 from R\&D systems.

\section{Bioinformatics and statistical analysis}

Data were analyzed with Microsoft Excel (Microsoft Co., Redmont, WA, USA), Prism 5 (GraphPad San Diego, CA, USA) and the environment R (R Foundation for Statistical Computing, Vienna, Austria). Data are presented as means \pm SEM and $p$-values were computed by paired or unpaired $t$-tests where applicable. Tumor growth was subjected to a linear mixed effect modeling applied to log pre-processed tumor volumes [61]. The $P$-values were calculated by testing jointly whether both tumor growth slopes and intercepts (on a log scale) are where dissimilar between treatment groups of interests. OS determined from the first injection and irRC at the date of diagnosis/time of treatment were used as the primary end points. Likelihood ratio test (LRT) from Cox proportional hazard regression modeling was used for assessing predictors of survival (including patient characteristics), as well as the added prognostic value of $\mathrm{SCD} 25$. All reported tests are twotailed and were considered significant for $P<0.05$. Regression models analyses were conducted on the log basis two transformed marker concentrations and were evaluated by means of Schoenfeld residuals. Therefore, hazard ratios and associated $95 \%$ confidence intervals are easily interpretable in terms of concentration doublings. For graphical representations, serum markers levels were partitioned into two out of three groups based on the observed median/tertile (sCD25, sLAG3) or a threshold of 250 (LDH).

\section{Acknowledgments}


We thank Philippe Rameau and Yann Lécluse for assistance in FACS and cell sorting experiments and Michelle Yong for help with mechanism studies in the mouse models. We are grateful to the staff of the animal facility of Gustave Roussy. DH was supported by ARC while l'Oreal awarded a prize to MV. GK and LZ were supported by the Ligue Nationale contre le Cancer (Equipes labellisées), SIRIC Socrates, Agence Nationale pour la Recherche (ANR AUTOPH, ANR Emergence), European Commission (ArtForce), European Research Council Advanced Investigator Grant (to GK), Fondation pour la Recherche Médicale (FRM), Institut National du Cancer (INCa), Fondation de France, Cancéropôle Ile-de-France, Fondation Bettencourt-Schueller, the LabEx Immuno-Oncology, the SIRIC Stratified Oncology Cell DNA Repair and Tumor Immune Elimination (SOCRATE), the SIRIC Cancer Research and Personalized Medicine (CARPEM), and the Paris Alliance of Cancer Research Institutes (PACRI). MJS was supported by a National Health and Medical Research Council (NH\&MRC) Australia Fellowship and Program Grant and a program grant from the Victorian Cancer Agency. MWLT was supported by a NH\&MRC Career Development Fellowship. MM was supported by the Associazione Italiana per la Ricerca sul Cancro.

\section{References}

1 Schreiber RD, Old LJ, Smyth MJ. Cancer immunoediting: integrating immunity's roles in cancer suppression and promotion. Science 2011; 331:1565-1570.

2 Fridman WH, Pages F, Sautes-Fridman C, Galon J. The immune contexture in human tumours: impact on clinical outcome. Nat Rev Cancer 2012; 12:298-306.

3 Bour-Jordan H, Esensten JH, Martinez-Llordella M, et al. Intrinsic and extrinsic control of peripheral T-cell tolerance by costimulatory molecules of the CD28/ B7 family. Immunol Rev 2011; 241:180-205.

4 Hodi FS, O'Day SJ, McDermott DF, et al. Improved survival with ipilimumab in patients with metastatic melanoma. $N$ Engl J Med 2010; 363:711-723.

5 Robert C, Thomas L, Bondarenko I, et al. Ipilimumab plus dacarbazine for previously untreated metastatic melanoma. $N$ Engl J Med 2011; 364:2517-2526.

6 Krummel MF, Allison JP. CD28 and CTLA-4 have opposing effects on the response of T cells to stimulation. $J$ Exp Med 1995; 182:459-465.

7 Peggs KS, Quezada SA, Allison JP. Cell intrinsic mechanisms of T-cell inhibition and application to cancer therapy. Immunol Rev 2008; 224:141-165.

8 Blair PJ, Riley JL, Levine BL, et al. CTLA-4 ligation delivers a unique signal to resting human CD4 T cells that inhibits interleukin-2 secretion but allows Bcl-X(L) induction. $J$ Immunol 1998; 160:12-15.

9 Chen W, Jin W, Wahl SM. Engagement of cytotoxic T lymphocyte-associated antigen 4 (CTLA-4) induces transforming growth factor beta (TGF-beta) production by murine CD4(+) T cells. J Exp Med 1998; 188:1849-1857.

10 Read S, Malmstrom V, Powrie F. Cytotoxic T lymphocyte-associated antigen 4 plays an essential role in the function of $\mathrm{CD} 25(+) \mathrm{CD} 4(+)$ regulatory cells that control intestinal inflammation. J Exp Med 2000; 192:295-302.

11 Wing K, Onishi Y, Prieto-Martin P, et al. CTLA-4 con- trol over Foxp3+ regulatory T cell function. Science 2008; 322:271-275.

12 Simpson TR, Li F, Montalvo-Ortiz W, et al. Fc-dependent depletion of tumor-infiltrating regulatory $\mathrm{T}$ cells co-defines the efficacy of anti-CTLA-4 therapy against melanoma. $J$ Exp Med 2013; 210:1695-1710.

13 Vanneman M, Dranoff G. Combining immunotherapy and targeted therapies in cancer treatment. Nat Rev Cancer 2012; 12:237-251.

14 Wada S, Jackson CM, Yoshimura K, et al. Sequencing CTLA4 blockade with cell-based immunotherapy for prostate cancer. J Transl Med 2013; 11:89.

15 Holmgaard RB, Zamarin D, Munn DH, Wolchok JD, Allison JP. Indoleamine 2,3-dioxygenase is a critical resistance mechanism in antitumor T cell immunotherapy targeting CTLA-4. $J$ Exp Med 2013; 210:1389-1402.

16 Lesterhuis WJ, Salmons J, Nowak AK, et al. Synergistic effect of CTLA-4 blockade and cancer chemotherapy in the induction of anti-tumor immunity. PLoS One 2013; 8: e61895.

17 Delyon J, Mateus C, Lefeuvre D, et al. Experience in daily practice with ipilimumab for the treatment of patients with metastatic melanoma: an early increase in lymphocyte and eosinophil counts is associated with improved survival. Ann Oncol 2013; 24:1697-1703.

18 Liakou CI, Kamat A, Tang DN, et al. CTLA-4 blockade increases IFNgamma-producing CD4+ICOShi cells to shift the ratio of effector to regulatory $\mathrm{T}$ cells in cancer patients. Proc Natl Acad Sci USA 2008; 105:14987-14992.

19 Maker AV, Attia P, Rosenberg SA. Analysis of the cellular mechanism of antitumor responses and autoimmunity in patients treated with CTLA-4 blockade. J Immunol 2005; 175:7746-7754.

20 Yuan J, Adamow M, Ginsberg BA, et al. Integrated NYESO-1 antibody and CD8+ T-cell responses correlate with clinical benefit in advanced melanoma patients treated with ipilimumab. Proc Natl Acad Sci USA 2011; 108:16723-16728.

21 Hodi FS, Butler M, Oble DA, et al. Immunologic and clinical effects of antibody blockade of cytotoxic T lymphocyte-associated antigen 4 in previously vaccinated cancer patients. Proc Natl Acad Sci USA 2008; 105:3005-3010.

22 Hamid O, Schmidt H, Nissan A, et al. A prospective phase II trial exploring the association between tumor microenvironment biomarkers and clinical activity of ipilimumab in advanced melanoma. J Transl Med 2011; 9:204.

23 Ribas A, Comin-Anduix B, Economou JS, et al. Intratumoral immune cell infiltrates, FoxP3, and indoleamine 2,3-dioxygenase in patients with melanoma undergoing CTLA4 blockade. Clin Cancer Res 2009; 15:390-399.

24 Chen H, Liakou CI, Kamat A, et al. Anti-CTLA-4 therapy results in higher CD4+ICOShi T cell frequency and IFN-gamma levels in both nonmalignant and malignant prostate tissues. Proc Natl Acad Sci USA 2009; 106:2729-2734.

25 Jinushi M, Hodi FS, Dranoff G. Therapy-induced antibodies to MHC class I chain-related protein A antagonize immune suppression and stimulate antitumor cytotoxicity. Proc Natl Acad Sci USA 2006; 103:9190-9195.

26 Ji RR, Chasalow SD, Wang L, et al. An immune-active tumor microenvironment favors clinical response to ipilimumab. Cancer Immunol Immunother 2012; 61:1019-1031. 
27 Yuan J, Zhou J, Dong Z, et al. Pretreatment serum VEGF is associated with clinical response and overall survival in advanced melanoma patients treated with Ipilimumab. Cancer Immunol Res 2014; 2:127-132.

28 Shrikant P, Khoruts A, Mescher MF. CTLA-4 blockade reverses CD8+ T cell tolerance to tumor by a CD4+ T cell- and IL-2-dependent mechanism. Immunity 1999; 11:483-493.

29 Maker AV, Phan GQ, Attia P, et al. Tumor regression and autoimmunity in patients treated with cytotoxic $\mathrm{T}$ lymphocyte-associated antigen 4 blockade and interleukin 2: a phase I/II study. Ann Surg Oncol 2005; 12:1005-1016.

30 Cabrera R, Ararat M, Eksioglu EA, et al. Influence of serum and soluble $\mathrm{CD} 25$ (sCD25) on regulatory and effector T-cell function in hepatocellular carcinoma. Scand J Immunol 2010; 72:293-301.

31 Gooding R, Riches P, Dadian G, Moore J, Gore M. Increased soluble interleukin-2 receptor concentration in plasma predicts a decreased cellular response to IL-2. Br J Cancer 1995; 72:452-455.

32 Eggermont AM, Suciu S, Testori A, et al. Long-term results of the randomized phase III trial EORTC 18991 of adjuvant therapy with pegylated interferon alfa- $2 \mathrm{~b}$ versus observation in resected stage III melanoma. J Clin Oncol 2012; 30:38103818.

33 Tourani JM, Pfister C, Tubiana N, et al. Subcutaneous interleukin-2 and interferon alfa administration in patients with metastatic renal cell carcinoma: final results of SCAPP III, a large, multicenter, phase II, nonrandomized study with sequential analysis design - the Subcutaneous Administration Propeukin Program Cooperative Group. J Clin Oncol 2003; 21:3987-3994.

34 Negrier S, Perol D, Ravaud A, et al. Randomized study of intravenous versus subcutaneous interleukin-2, and IFNalpha in patients with good prognosis metastatic renal cancer. Clin Cancer Res 2008; 14:5907-5912.

35 Lotze MT, Matory YL, Ettinghausen SE, et al. In vivo administration of purified human interleukin 2. II. Half life, immunologic effects, and expansion of peripheral lymphoid cells in vivo with recombinant IL 2. J Immunol 1985; 135:2865-2875.

36 Okamura T, Fujio K, Sumitomo S, Yamamoto K. Roles of LAG3 and EGR2 in regulatory T cells. Ann Rheum Dis 2012; 71 Suppl 2:i96-i100.

37 Saadoun D, Rosenzwajg M, Joly F, et al. Regulatory T-cell responses to low-dose interleukin-2 in HCV-induced vasculitis. N Engl J Med 2011; 365:2067-2077.

38 Bien E, Balcerska A. Serum soluble interleukin 2 receptor alpha in human cancer of adults and children: a review. Biomarkers 2008; 13:1-26.

39 Kavanagh B, O'Brien S, Lee D, et al. CTLA4 blockade expands FoxP3+ regulatory and activated effector CD4+ T cells in a dose-dependent fashion. Blood 2008; 112:1175-1183.

40 Balch CM, Gershenwald JE, Soong SJ, et al. Final version of 2009 AJCC melanoma staging and classification. J Clin Oncol 2009; 27:6199-6206.

41 Mellman I, Coukos G, Dranoff G. Cancer immunotherapy comes of age. Nature 2011; 480:480-489.

42 Waterhouse P, Penninger JM, Timms E, et al. Lymphoproliferative disorders with early lethality in mice deficient in Ctla4. Science 1995; 270:985-988.
43 Quezada SA, Peggs KS, Curran MA, Allison JP. CTLA4 blockade and GM-CSF combination immunotherapy alters the intratumor balance of effector and regulatory T cells. $J$ Clin Invest 2006; 116:1935-1945.

44 Hannier S, Tournier M, Bismuth G, Triebel F. CD3/TCR complex-associated lymphocyte activation gene-3 molecules inhibit CD3/TCR signaling. J Immunol 1998; 161:4058-4065.

45 Gagliani N, Magnani CF, Huber S, et al. Coexpression of CD49b and LAG-3 identifies human and mouse T regulatory type 1 cells. Nat Med 2013; 19:739-746.

46 Zheng Y, Zha Y, Driessens G, Locke F, Gajewski TF. Transcriptional regulator early growth response gene 2 (Egr2) is required for T cell anergy in vitro and in vivo. $J$ Exp Med 2012; 209:2157-2163.

47 Lotze MT, Custer MC, Sharrow SO, et al. In vivo administration of purified human interleukin-2 to patients with cancer: development of interleukin-2 receptor positive cells and circulating soluble interleukin-2 receptors following interleukin-2 administration. Cancer Res 1987; 47:2188-2195.

48 Boyano MD, Garcia-Vazquez MD, Lopez-Michelena T, et al. Soluble interleukin-2 receptor, intercellular adhesion molecule-1 and interleukin-10 serum levels in patients with melanoma. Br J Cancer 2000; 83:847-852.

49 Lissoni P, Tancini G, Rovelli F, et al. Serum interleukin-2 levels in relation to the neuroendocrine status in cancer patients. Br J Cancer 1990; 62:838-839.

50 Nakase K, Tsuji K, Tamaki S, et al. Elevated levels of soluble interleukin-2 receptor in serum of patients with hematological or non-hematological malignancies. Cancer Detect Prev 2005; 29:256-259.

51 Ottaiano A, Leonardi E, Simeone E, et al. Soluble interleukin-2 receptor in stage I-III melanoma. Cytokine 2006; 33:150-155.

52 Sabbioni ME, Siegrist HP, Bacchi M, et al. Association between immunity and prognostic factors in early stage breast cancer patients before adjuvant treatment. Breast Cancer Res Treat 2000; 59:279-287.

53 Maier LM, Anderson DE, Severson CA, et al. Soluble IL-2RA levels in multiple sclerosis subjects and the effect of soluble IL-2RA on immune responses. J Immunol 2009; 182:15411547.

54 Symons JA, Wood NC, Di Giovine FS, Duff GW. Soluble IL-2 receptor in rheumatoid arthritis. Correlation with disease activity, IL-1 and IL-2 inhibition. J Immunol 1988; 141:26122618.

55 Zorn U, Dallmann I, Grosse J, et al. Soluble interleukin 2 receptors abrogate IL-2 induced activation of peripheral mononuclear cells. Cytokine 1994; 6:358-364.

56 Russell SE, Moore AC, Fallon PG, Walsh PT. Soluble IL-2Ralpha (sCD25) exacerbates autoimmunity and enhances the development of Th17 responses in mice. PLoS One 2012; 7:e47748.

57 Wilgenhof S, Du Four S, Vandenbroucke F, et al. Single-center experience with ipilimumab in an expanded access program for patients with pretreated advanced melanoma. $J$ Immunother 2013; 36:215-222.

58 Kelderman S, Heemskerk B, van Tinteren H, et al. Lactate dehydrogenase as a selection criterion for ipilimumab treatment in metastatic melanoma. Cancer Immunol Immunother 2014; 
63:449-458.

59 Morris JC, Waldmann TA. Advances in interleukin 2 receptor targeted treatment. Ann Rheum Dis 2000; 59 Suppl 1:i109-i114.

60 Peggs KS, Quezada SA, Chambers CA, Korman AJ, Allison JP. Blockade of CTLA-4 on both effector and regulatory $\mathrm{T}$ cell compartments contributes to the antitumor activity of anti-CTLA-4 antibodies. J Exp Med 2009; 206:1717-1725.

61 Sugar E, Pascoe AJ, Azad N. Reporting of preclinical tumor-graft cancer therapeutic studies. Cancer Biol Ther 2012; 13:1262-1268.

(Supplementary information is linked to the online version of the paper on the Cell Research website.) 